\title{
Omnipose: a high-precision morphology-independent solution for bacterial cell segmentation
}

\author{
Kevin J. Cutler ${ }^{1}$, Carsen Stringer ${ }^{2}$, Paul A. Wiggins ${ }^{1,3,{ }^{*}}$ and Joseph D. Mougous ${ }^{4,5, *}$
}

${ }^{1}$ Department of Physics, University of Washington, Seattle, WA 98195, USA

${ }^{2}$ HHMI Janelia Research Campus, Ashburn, VA, USA

${ }^{3}$ Department of Bioengineering, University of Washington, Seattle, WA 98195, USA

${ }^{4}$ Department of Microbiology, University of Washington, Seattle, WA 98109, USA

${ }^{5}$ Howard Hughes Medical Institute, University of Washington, Seattle, WA 98195, USA

* To whom correspondence should be addressed:

Email-pwiggins@uw.edu,mougous@uw.edu 


\section{Abstract}

Advances in microscopy hold great promise for allowing quantitative and precise

3 readouts of morphological and molecular phenomena at the single cell level in bacteria.

4 However, the potential of this approach is ultimately limited by the availability of

5 methods to perform unbiased cell segmentation, defined as the ability to faithfully

6 identify cells independent of their morphology or optical characteristics. In this study, we

7 present a new algorithm, Omnipose, which accurately segments samples that present

8 significant challenges to current algorithms, including mixed bacterial cultures,

9 antibiotic-treated cells, and cells of extended or branched morphology. We show that

10 Omnipose achieves generality and performance beyond leading algorithms and its

11 predecessor, Cellpose, by virtue of unique neural network outputs such as the gradient of

12 the distance field. Finally, we demonstrate the utility of Omnipose in the characterization

13 of extreme morphological phenotypes that arise during interbacterial antagonism and on

14 the segmentation of non-bacterial objects. Our results distinguish Omnipose as a uniquely

15 powerful tool for answering diverse questions in bacterial cell biology. 


\section{Introduction}

17 Although light microscopy is a valuable tool for characterizing cellular and sub-

18 cellular structures and dynamics, quantitative analysis of microscopic data remains a

19 persistent challenge ${ }^{1}$. This is especially pertinent to the study of bacteria, many of which

20 have dimensions in the range of optical wavelengths. Thus, their cell body is composed

21 of a small number of pixels (e.g., $\sim 100-300 \mathrm{px}^{2}$ for E. coli at $100 \mathrm{x}$ magnification). At this

22 scale, accurate subcellular localization requires defining the cell boundary with single-

23 pixel precision. The process of defining cell boundaries within micrographs is termed cell

24 segmentation and this is a critical first step in current image analysis pipelines ${ }^{2,3}$.

25 In addition to their small size, bacteria adopt a wide range of morphologies.

26 Although many commonly studied bacteria are well-approximated by idealized rods or

27 spheres, there is growing interest in bacteria with more elaborate shapes ${ }^{4}$. Some examples

28 include Streptomycetales, which form long filamentous and branched hyphal structures ${ }^{5}$,

29 and Caulobacterales, which generate extended appendages distinct from their cytoplasm ${ }^{6}$.

30 Furthermore, microfluidic devices are allowing researchers to capture the responses of

31 bacteria to assorted treatments such as antibiotics, which often result in highly irregular

32 morphologies ${ }^{7}$. Whether native or induced, atypical cell morphologies present a distinct

33 problem at the cell segmentation phase of image analysis ${ }^{8,9}$. This is compounded when

34 such cells are present with those adopting other morphologies, as is the case in many

35 natural samples of interest ${ }^{10}$. To date, there are no solutions for segmenting bacterial cells

36 of assorted shape and size in a generalizable manner ${ }^{1}$.

37 Cell segmentation is a complex problem that extends beyond microbiological

38 research, thus many solutions are currently available in image analysis programs ${ }^{8,9,11-27}$. 
39 Most of these solutions use traditional image processing techniques such as the

40 application of an intensity threshold to segment isolated cells; however, this approach

41 does not perform well for cells in close contact and it requires extensive parameter-tuning

42 in order to optimize for a given cell type. SuperSegger was developed to address these

43 issues specifically in bacterial phase contrast images ${ }^{13}$. This program utilizes both

44 traditional image filtering techniques and a shallow neural network to correct for errors

45 that thresholding and watershed segmentation tend to produce.

46 Deep neural networks (DNNs) are now widely recognized as superior tools for

47 cell segmentation ${ }^{28}$. Unlike traditional image processing, machine-learning approaches

48 such as DNNs require training on a ground-truth dataset of cells and corresponding

49 labels. Trained DNNs are thus limited in applicability to images that are representative of

50 those in the training dataset. Early DNN approaches were based on the Mask R-CNN

51 architecture ${ }^{24}$, whereas more recent algorithms such as StarDist, Cellpose, and MiSiC are

52 based on the U-Net architecture ${ }^{12,15,26}$. Pachitariu and colleagues showed that Cellpose

53 outperforms Mask R-CNN and StarDist on a variety of cell types and cell-like objects,

54 distinguishing it as a general solution for cell segmentation ${ }^{12}$. Notably, the representation

55 of bacteria in their study was limited. MiSiC was developed as a general DNN-based

56 solution for bacterial segmentation; however, the authors of MiSiC did not provide

57 comparisons to other DNN algorithms ${ }^{15}$. Here, we evaluated the performance of state-of-

58 the-art cell segmentation algorithms on a diverse collection of bacterial cells. Our

59 findings motivated the design of a new algorithm, Omnipose, that significantly

60 outperforms all previous cell segmentation algorithms across a wide range of bacterial

61 cell sizes, morphologies, and optical characteristics. We have made Omnipose and all 
62 associated data immediately available to researchers, and we anticipate that our model -

63 without retraining - can be applied to diverse bacteriological systems. Furthermore,

64 following the incorporation of additional ground truth data, Omnipose could serve as a

65 platform for segmenting various eukaryotic cells and extended, anisotropic objects more

66 broadly.

68 Results

69 Evaluation of bacterial cell segmentation algorithms

70 Numerous image segmentation algorithms have been developed, and the

71 performance of many of these on bacterial cells is documented ${ }^{1}$. These broadly fall into

72 three categories: $(i)$ traditional image processing approaches (e.g., thresholding,

73 watershed), (ii) traditional/machine learning hybrid approaches, and (iii) deep neural

74 network (DNN) approaches. Given the goal of developing software with the capacity to

75 recognize bacteria universally, we sought to identify strongly performing algorithms for

76 further development. An unbiased, quantitative comparison of cell segmentation

77 algorithms on bacterial cells has not been performed; thus, we selected one or more

78 representatives from each category for our analysis: Morphometrics ${ }^{23}(i), \operatorname{SuperSegger}^{13}$

79 (ii), Mask R-CNN ${ }^{27}$, StarDist $^{26}, \mathrm{MiSiC}^{15}$, and Cellpose ${ }^{12}$ (iii). For a detailed review of

80 these choices, see Methods.

81 For training and benchmarking these algorithms, we acquired micrographs of

82 assorted bacterial species representing diverse morphologies and optical characteristics.

83 Many studies of bacteria involve mutations or treatments that cause extreme

84 morphologies. To capture this additional diversity, we included wild-type and mutant 
85 bacteria grown in the presence of two beta-lactam antibiotics, cephalexin and aztreonam,

86 and A22, which targets $\mathrm{MreB}^{29}$. Finally, based on our interest in microbial communities,

87 we acquired images of mixtures of bacteria which display distinct morphologies and

88 optical characteristics. In total, we collected 4833 images constituting approximately

89700,900 individual cells deriving from 14 species (Extended Data Table 1). Next, we

90 developed a streamlined approach for manual cell annotation and applied it to these

91 images (see Methods), yielding 46,000 representative annotated cells that serve as our

92 ground-truth dataset. We arbitrarily split this data into a 27,000-cell training set and a

93 19,000-cell benchmarking set. Relevant cellular metrics did not differ substantially

94 between the groups, confirming that the benchmarking set faithfully represents the

95 training set (Extended Data Fig. 1).

96 To facilitate direct comparison of the algorithms, we first optimized their

97 performance against our data. For the DNN approaches, each algorithm was trained on

98 our dataset using developer-recommended parameters. Morphometrics and SuperSegger

99 cannot be automatically optimized using ground-truth data; therefore, we manually

100 identified settings that optimized the performance of these algorithms against our dataset

101 (see Methods). As a quantitative measure for algorithm performance, we compared their

102 average Jaccard Index (JI) as a function of intersection over union (IoU) threshold - a

103 well-documented approach for evaluating image segmentation (Fig. 1a) ${ }^{30,31}$. IoU values

104 lie between zero and one, with values greater than 0.8 marking the point at which masks

105 become indistinguishable from ground truth by the expert human eye (Extended Data

106 Fig. 2) $)^{30}$. This analysis showed that DNN-based approaches significantly outperform

107 other algorithms. However, within the DNN group, substantial differences in 
108 performance were observed; Cellpose and StarDist outperform Mask R-CNN and MiSiC

109 at high IoU thresholds. The performance of all algorithms varied greatly across the

110 images in our ground-truth dataset, with much of this variability delineated by cell type

111 and morphology categories (Fig. 1b-g). Whereas all other algorithms exhibited visible

112 segmentation errors in two of the three cell categories we defined, errors by Cellpose -

113 the best overall performing algorithm at high IoU thresholds - were only apparent in

114 elongated cells (Fig. 1h-j).

\section{Motivation for a new DNN-based segmentation algorithm}

117 Our comparison revealed that Cellpose offers superior performance relative to the

118 other segmentation algorithms we analyzed, and for this reason, we selected this

119 algorithm for further development. Notably, even at the high performance levels of

120 Cellpose, only $83 \%$ of predictions on our benchmarking dataset are above 0.8 IoU. This

121 limits the feasibility of highly quantitative studies such as those involving subcellular

122 protein localization or cell-cell interactions.

123 Cellpose utilizes a neural network that is trained on ground-truth examples to

124 transform an input image into several intermediate outputs, including a scalar probability

125 field for identifying cell pixels (Extended Data Fig. 3a, panels $i$-iii) ${ }^{12}$. Cellpose is unique

126 among DNN algorithms by the addition of a vector field output, which is defined by the

127 normalized gradient of a heat distribution from the median cell pixel coordinate

128 (Extended Data Fig. 3a, panels $i v, v$ ). This vector field directs pixels toward a global cell

129 center via Euler integration, allowing cells to be segmented based on the points at which

130 pixels coalesce (Extended Data Fig. 3b). In contrast to other algorithms, this approach for 
131 reconstructing cells is size- and morphology-independent, insofar as the cell center can be

132 correctly defined.

133 To further interrogate the accuracy of Cellpose on our dataset, we evaluated its

134 performance as a function of cell size. We compared cell area against the number of

135 segmentation errors, calculated as the number of redundant or missing masks

136 corresponding to each ground-truth cell mask. This revealed a strong correlation between

137 cell size and segmentation errors, with the top quartile of cells accounting for $83 \%$ of all

138 errors (Fig. 2a). To understand the source of these errors, we inspected the flow field

139 output of many poorly segmented cells across a variety of species and growth conditions.

140 This showed that elongated cells, an important morphology often seen in both wild-type

141 and mutant bacterial populations, are particularly susceptible to over-segmentation (Fig.

142 2b). We attribute this to the multiple sinks apparent in the corresponding flow fields. In

143 the Cellpose mask reconstruction algorithm, pixels belonging to these cells are guided

144 into multiple centers per cell, fragmenting the cell into many separate masks.

145 We hypothesized that the observed defect in Cellpose flow field output is a

146 consequence of two distinct flow field types arising from our training dataset: those

147 where the median pixel coordinate, or 'center', lies within the cell $(97.8 \%)$ and those

148 where it lies outside the cell $(2.2 \%)$. In the latter, Cellpose projects the center point to the

149 nearest boundary pixel, ultimately leading to points of negative divergence on the cell

150 periphery that are chaotically distributed (Fig. 2c-e). On the contrary, non-projected

151 centers maintain a uniform field magnitude along the entire boundary and adhere to the

152 global symmetries of the cell (Extended Data Fig. 4a,d). A similar issue is also

153 encountered in cells with centers that are not projected but lie close to the boundary 
154 (Extended Data Fig. 4b-d). Cells with a center point closer than 0.3 times the mean cell

155 diameter (a factor of 0.2 off-center) to the boundary account for an additional $8.5 \%$ of our

156 data. Neural networks can be exquisitely sensitive to the outliers in their training data ${ }^{32}$;

157 therefore, we suspect that this small fraction of corrupt flow fields has significantly

158 impacted the performance of Cellpose.

As there exists no straightforward means of defining a cell center for irregular

162 objects, we sought to develop a segmentation algorithm that operates independently of

163 cell center identification. We built our new algorithm, which we named Omnipose,

164 around the scalar potential known as the distance field (or distance transform), which

165 describes the distance at any point $\vec{x}$ in a bounded region $\Omega$ to the closest point on the

166 boundary $\partial \Omega$. Notably, this widely utilized construct is one of the intermediate outputs of

167 StarDist ${ }^{32}$. Whereas in StarDist it is used to seed and assemble star-convex polygons, its

168 use in Omnipose is to define a new flow field within the Cellpose framework. The use of

169 a distance field has several advantages. First, the distance field is defined by the eikonal

170 equation $|\vec{\nabla} \Phi(\vec{x})|=1$, and so its gradient has unit magnitude throughout the bounded

171 region for which it is calculated. This grants it faster convergence and better numerical

172 stability when compared to alternative solutions producing similar fields (e.g., screened

173 Poisson; see Methods) (Extended Data Fig. 5a). Second, the distance field is independent

174 of morphology and topology, meaning that it is applicable to all cells. Lastly, the

175 resulting flow field points uniformly from cell boundaries toward the local cell center,

176 coinciding with the medial axis, or skeleton, that is defined by the stationary points of the 
177 distance field (Extended Data Fig. 5b). This critical feature allows pixels to remain

178 spatially clustered after Euler integration, solving the problem of over-segmentation seen

179 in Cellpose.

180 One challenge to using the distance field as the basis to our approach is that

181 traditional distance field algorithms like FMM (Fast Marching Method) are sensitive to

182 boundary pixilation ${ }^{33}$, causing artifacts in the flow field that extend deep into the cell.

183 These artifacts are sensitive to pixel-scale changes at the cell perimeter, which we

184 reasoned would interfere with the training process. To solve this, we developed an

185 alternative approach based on FIM (Fast Iterative Method) that produces smooth distance

186 fields for arbitrary cell shapes and sizes (Fig. 3a, and see Methods) ${ }^{34}$. The corresponding

187 flow field is relatively insensitive to boundary features at points removed from the cell

188 boundary, a critical property for robust and generalized prediction by the Cellpose

189 network.

190 The use of the distance field additionally required a unique solution for mask

191 reconstruction. Whereas the pixels in a center-seeking field converge on a point, standard

192 Euler integration under our distance-derived field tends to cluster pixels into multiple thin

193 fragments along the skeleton, causing over-segmentation (Fig. 3b). We solved this with a

194 suppression factor of $(t+1)^{-1}$ in each time step of the Euler integration. This reduces

195 the movement of each pixel after the first step $t=0$, facilitating initial cell separation

196 while preventing pixels from clustering into a fragmented skeleton formation. The wider

197 distribution resulting from our suppression factor allows pixels to remain connected,

198 thereby generating a single mask for each cell in conjunction with a standard automated

199 pixel clustering algorithm (e.g., DBSCAN $)^{35}$. 


\section{Omnipose demonstrates unprecedented segmentation accuracy of bacterial cells}

With solutions to the major challenges of cell center-independent segmentation

203 incorporated into Omnipose, we proceeded to benchmark its performance. Remarkably,

204 across the IoU threshold range 0.5-1.0, Omnipose averages a JI $>10$-fold above that of

205 Cellpose (Fig. 4a). The difference in performance between the algorithms is particularly

206 pronounced within the high IoU range (0.75-1.0), where we observe an average of 170-

207 fold higher JI for Omnipose. At the $0.5-5 \mu \mathrm{m}$ scale and with a typical microscope

208 configuration, quantitative measurements rely upon IoU values in this range, thus

209 Omnipose is uniquely suited for the microscopic analysis of bacterial cells.

210 To dissect the contributions of the individual Omnipose innovations to the overall

211 performance of the algorithm, we isolated the mask reconstruction component of

212 Omnipose and applied it to the Cellpose network output. This augmentation of Cellpose

213 modestly improved its performance to a roughly equivalent extent across all IoU

214 thresholds (Fig. 4a). Based on this, we attribute the remaining gains in performance by

215 Omnipose to its unique network outputs and our improvements to the Cellpose training

216 framework (see Methods).

217 Our analyses illuminated critical flaws in prior DNN-based approaches for the

218 segmentation of elongated cells, effectively preventing these algorithms from

219 generalizable application to bacteria (Fig. 1). To determine whether Omnipose overcomes

220 this limitation, we evaluated its performance as a function of cell area. Cell area serves as

221 a convenient proxy for cell length in our dataset, which is composed of both branched

222 and unbranched elongated cells. Whereas the Cellpose cell error rate remains above 9\% 
223 and increases exponentially with cell size, Omnipose displays a consistent error rate that

224 remains below 4\% for all percentiles (Fig. 4b). Thus, Omnipose performance is

225 independent of cell size and shape, including those cells with complex, extended

226 morphologies (Fig. 4c,d).

228 Omnipose permits sensitive detection of cellular intoxication

230 delivered toxin produced by Serratia proteamaculans, Tre ${ }^{36}$. We showed that this toxin

231 acts by ADP-ribosylating the essential cell division factor FtsZ; however, we were unable

232 to robustly evaluate the consequences of Tre1 intoxication on target cell morphology

233 owing to segmentation challenges. Here we asked whether Omnipose could permit

234 straightforward and sensitive detection of intoxication by Tre1. To this end, we incubated

235 S. proteamaculans wild-type or a control strain expressing inactive Tre1 (tre $1^{E 415 Q}$ ) with

236 target E. coli cells and imaged these mixtures after a fixed period of 20 hours. Owing to

237 the unique capabilities of Omnipose, we were able to include dense fields of view,

238 incorporating $>300,000$ cells in our analysis.

239 Among the cells identified by Omnipose, we found a small proportion were

240 elongated and much larger than typical bacteria (Fig. 5a,b and Extended Data Fig. 6a).

241 These cells were only detected in mixtures containing active Tre1, and the apparent

242 failure of the cells to septate is consistent with the known FtsZ-inhibitory activity of the

243 toxin. The $S$. proteamaculans strain background we employed in this work expresses the

244 green fluorescent protein. Corresponding fluorescence images allowed us to

245 unambiguously assign the enlarged cell population to E. coli (Fig. 5c). Next, we 
246 subjected the same images to cell segmentation with StarDist, Cellpose, and MiSiC, the

247 three top-performing algorithms in our initial survey. Each of these algorithms fail to

248 identify this population of cells to high precision (Fig. 5d,e). Close inspection reveals

249 three distinct modes of failure (Fig. 5e and Extended Data Fig. 6b). In the case of

250 StarDist, elongated (non-star-convex) cells are split into multiple star-convex subsets that

251 do not span the entire cell. Cellpose detects entire elongated cells, but it breaks them up

252 into a multitude of smaller masks. Conversely, MiSiC detects all cells but fails to

253 properly separate them, thereby exaggerating the area measurement in many cases. Taken

254 together, these data illustrate how the enhanced cell segmentation performance of

255 Omnipose can facilitate unique insights into microbiological systems.

257 Omnipose exhibits strong performance in non-bacterial segmentation tasks

258 We have shown that the features we developed within Omnipose improved phase-

259 contrast bacterial segmentation performance beyond that of Cellpose. However, it is

260 possible that these features could hinder performance relative to Cellpose in other

261 imaging modalities or in segmentation tasks involving, for example, eukaryotic cells,

262 whole organisms, and cell-like objects. To test this, we trained Omnipose on the cyto2

263 dataset, a large collection of images and corresponding ground-truth annotations

264 submitted by users that expands upon the original cyto dataset of Cellpose ${ }^{12,30}$. We found

265 that Omnipose offers a modest improvement in performance relative to Cellpose on this

266 dataset (Fig. $5 f$ and Extended Data Fig. 7a). Moreover, Omnipose achieved this

267 performance boost without compromising the segmentation rate ( $\sim 1$ image per second). 
269 sought to investigate its potential utility in a field far removed from microbiology. The

270 nematode Caenorhabditis elegans is a widely studied model organism with an overall

271 morphology grossly similar to elongated bacteria ${ }^{37}$. At just one millimeter in length,

272 intact C. elegans are often analyzed by microscopy in order to measure phenotypes;

273 therefore, there is significant interest in methods for their accurate segmentation to enable

274 tracking ${ }^{38}$. We obtained, annotated and trained Omnipose on two publicly available

275 microscopy datasets composed of C. elegans images: time-lapse frames from the Open

276 Worm Movement database ${ }^{39}$ and frames containing fields of assorted live or dead $C$.

277 elegans from the $\mathrm{BBBC} 010$ dataset $^{40}$. These images contain debris and are of

278 heterogenous quality, yet $82 \%$ of masks predicted by Omnipose match or exceed the 0.8

279 IoU threshold (Fig. 5g,h). Taken together with our findings on cyto2, we conclude that

280 Omnipose inherits and offers improvement upon the broad applicability of Cellpose.

\section{Discussion}

283 Confronted with the importance of segmentation accuracy to the success of work

284 within our own laboratory, we were motivated to characterize the performance of several

285 existing cell segmentation algorithms. Recent developments in deep learning have greatly

286 improved these algorithms; however, significant challenges remain ${ }^{1,30}$. Although isolated

287 cells without cell-to-cell contact can be segmented with high precision by any of the

288 packages we tested, segmentation becomes significantly more challenging when cells

289 form microcolonies, adopt irregular morphologies, or when fields are composed of cells

290 with multiple shapes and sizes. Such difficulties are compounded in time-lapse studies, 
291 where the significance of segmentation errors often grows exponentially with time.

292 Experimental design can help mitigate certain segmentation challenges; however, the

293 recent emphasis on non-model organisms and microbial communities renders this an

294 increasingly undesirable solution ${ }^{41}$.

295 This work provides the most comprehensive side-by-side quantitative comparison

296 of cell segmentation algorithm performance to-date. As expected, machine-learning-

297 based approaches outperform others, yet insights into general image segmentation

298 strategies can be gained from each of the methods we examined. Two of the six

299 algorithms we tested utilize traditional image thresholding and watershed segmentation:

300 Morphometrics and SuperSegger ${ }^{13,23}$. Each program tends to under-segment adjacent

301 cells and over-segment large cells, behaviors previously linked to thresholding and

302 watershed processes, respectively ${ }^{1,42}$. Given that SuperSegger was motivated at least in-

303 part to mitigate these issues, we postulate that traditional image segmentation approaches

304 are ultimately limited to specialized imaging scenarios. Although we classify MiSiC as a

305 DNN-based approach, this algorithm also relies on thresholding and watershed

306 segmentation to generate cell masks from its network output ${ }^{15}$. The network output of

307 MiSiC is more uniform than unfiltered phase contrast images, yet this pre-processing

308 does not fully abrogate the typical errors of thresholding and watershed segmentation.

309 We therefore conclude that, even when combined with neural networks as seen in MiSiC,

310 thresholding and watershed cannot be effectively used for general cell segmentation

311 tasks.

312 A successful DNN-based algorithm is composed of a robust, consistent neural

313 network output, and an appropriate mask reconstruction process based on this output. In 
314 the case of Mask R-CNN, bounding boxes for each cell are predicted along with a

315 probability field that localizes a cell within its bounding box ${ }^{43}$. Masks are generated by

316 iterating over each box and thresholding the probability field. Despite the widespread

317 adoption of Mask R-CNN, we found this algorithm did not perform exceptionally well in

318 our study. Our results suggest that this is due to dense cell fields with overlapping

319 bounding boxes, a feature known to corrupt the training process and produce poor

320 network outputs for Mask R-CNN ${ }^{44}$. By contrast, the StarDist network makes robust

321 predictions, but it fails to assemble accurate cell masks because the cells in our dataset

322 are not well approximated by star-convex polygons ${ }^{26}$. The errors we encountered with

323 Cellpose can be attributed to both neural network output and mask reconstruction. In

324 Omnipose, we specifically addressed these two issues via the distance field and

325 suppressed Euler integration, respectively, yielding a remarkably precise and

326 generalizable image segmentation tool. Omnipose effectively leverages the strongest

327 features of several of the DNN approaches we tested, namely the distance field of

328 StarDist, the boundary field of MiSiC, and the mask reconstruction framework of

329 Cellpose.

330 We have designed Omnipose for use by typical research laboratories and we have

331 made its source code and training data publicly available. For images of bacteria under

332 phase contrast, researchers will not need to provide new ground truth data or retrain the

333 model. In this study, we emphasized morphological diversity, but we further accounted

334 for differences in optical features between bacterial strains, slide preparation techniques,

335 and microscope configurations. For example, the images in our ground-truth dataset

336 originate from four different researchers using distinct microscopes, objectives, sensors, 
337 illumination sources, and acquisition settings. We further introduced extensive image

338 augmentations that simulate variations in image intensity, noise, gamma, clipping, and

339 magnification. Lastly, bacterial strains exhibit a wide range of intrinsic contrast and

340 internal structure, often exacerbated by antibiotic treatment or revealed by dense cell

341 packing. Internal structure can cause over-segmentation, so we included many cells with

342 this characteristic in our dataset.

343 We anticipate that the unprecedented performance of Omnipose may permit

344 access to information from microscopy images that was previously inaccessible. For

345 instance, images deriving from natural microbial communities could be accurately

346 characterized with regard to internal structure, autofluorescence, and morphology at the

347 single-cell level. This data could be used to estimate diversity, a novel methodology that

348 would complement existing sequencing-based metrics ${ }^{45}$. It is worth noting that

349 phenotypic diversity often exceeds genetic diversity ${ }^{46}$; therefore, even in a relatively

350 homogeneous collection of organisms, precise segmentation could allow classes

351 representing distinct states to be identified. A microscopy-based approach also offers the

352 opportunity to characterize spatial relationships between cells, information that is

353 exceptionally difficult to recover in most biomolecular assays. 


\section{Methods}

\section{Phase contrast and fluorescence microscopy}

356 In-house imaging was performed on a Nikon Eclipse Ti-E wide-field epi-

357 fluorescence microscope, equipped with a sCMOS camera (Hamamatsu) and X-cite LED

358 for fluorescence imaging. We imaged through 60X and 100X 1.4 NA oil-immersion PH3

359 objectives. The microscope was controlled by NIS-Elements. Cell samples were spotted

360 on a $3 \%(\mathrm{w} / \mathrm{v})$ agarose pad placed on a microscope slide. The microscope chamber was

361 heated to $30^{\circ} \mathrm{C}$ or $37^{\circ} \mathrm{C}$ when needed for time-lapse experiments.

362 Several images in our dataset were taken by two other laboratories using three

363 distinct microscope/camera configurations. The Brun lab provided images of $C$.

364 crescentus acquired on a Nikon Ti-E microscope equipped with a Photometrics Prime

365 95B sCMOS camera. Images were captured through a 60X Plan Apo $\lambda$ 100X 1.45 NA oil

366 Ph3 DM objective. The Wiggins lab provided E. coli and A. baylyi time lapses from both

367 a Nikon Ti-E microscope as well as a custom-built tabletop microscope, both described in 368 previous studies ${ }^{47,48}$.

370 C. elegans data preparation

371 We obtained a 1000-frame time lapse of C. elegans from the Wormpose ${ }^{38}$ GitHub

372 (https://github.com/iteal/wormpose_data) adapted from the Open Worm Movement

373 database ${ }^{39}$, which is inaccessible at the time of writing. We also utilized the Broad

374 Bioimage Benchmarking Collection set BBBC010 ${ }^{40}$ (https://bbbc.broadinstitute.org/c-

375 elegans-livedead-assay-0), a set of 100 images containing live and dead C. elegans.

376 These images were manually cropped to select regions of each image without C. elegans 
377 overlaps. For both of these datasets, images were initially segmented with Omnipose to

378 select foreground, automatically cropped to select individual C. elegans or clusters of $C$.

379 elegans, and then packed into ensemble images for efficient annotation, training, and

380 testing following the same procedures described below for our bacterial datasets.

\section{Bacterial sample preparation}

383 To image antibiotic-induced phenotypes, cells were grown without antibiotics

384 overnight in LB, back-diluted, and spotted on agarose pads with $50 \mu \mathrm{g} / \mathrm{mL}$ A22 or

$38510 \mu \mathrm{g} / \mathrm{mL}$ cephalexin. Time lapses were captured of E. coli DH5 $\alpha$ and S. flexneri M90T

386 growing on these pads. E. coli CS703-1 was back-diluted into LB containing $1 \mu \mathrm{g} / \mathrm{mL}$

387 aztreonam and spotted onto a pad without antibiotics ${ }^{49}$. Cells constitutively expressed

388 GFP to visualize cell boundaries.

389 H. pylori LSH100 grown with and without Aztreonam was provided by the

390 Salama $1 \mathrm{ab}^{50,51}$. Samples were fixed and stained with Alexaflour 488 to visualize the cell

391 membrane. Images were taken on LB pads. The typical technique of allowing the spot to

392 dry on the pad caused cells to curl up on themselves, so our images were taken by placing

393 the cover slip on the pad immediately after spotting and applying pressure to force out

394 excess media.

395 C. crescentus was cultivated and imaged by the Brun lab ${ }^{52,53}$. Cells were grown in

396 PYE, washed twice in water prior to 1:20 dilution in Hutner base-imidazole-buffered-

397 glucose-glutamate (HIGG media) and grown at $26^{\circ} \mathrm{C}$ for $72 \mathrm{~h}$. Cells were spotted on a $1 \%$

398 agarose PYE pads prior to imaging. 
400 Yeast extract 4g/L, Malt extract 10g/L, Dextrose 4g/L, Agar 20g/L. This media was used

401 to first culture the bacteria in liquid overnight and then on a pad under the microscope.

402 This strain forms aggregates in liquid media, so these aggregates were allowed to grow

403 for several hours on a slide in the heated microscope chamber until we could see

404 individual filaments extending from the aggregates. Fields of view were selected and

405 cropped to exclude cell overlaps. Autofluorescence was captured to aid in manual

406 segmentation.

407 Mixtures of $S$. proteamaculans attTn $7::$ Km-gfp trel or tre $1^{E 415 Q}$ and E. coli were

408 spotted on a PBS pad to prevent further growth. Phase-contrast images of the cells were

409 acquired before and after a $20 \mathrm{hr}$ competition on a high-salt LB plate. Fluorescence

410 images in the GFP channel were also acquired to distinguish S. proteamaculans from

411 unlabeled E. coli.

412 All other individual strains in Table S1 were grown overnight, diluted 1:100 into

413 fresh LB media, and grown for 1-3 hours before imaging. Mixtures were made by

414 combining back-diluted cells roughly 1:1 by $\mathrm{OD}_{600}$.

416 Manual image annotation

417 Manual annotation began with loading images into MATLAB, normalizing the

418 channels, registering the fluorescence channel(s) to brightfield (when applicable), and

419 producing boundary-enhanced versions of brightfield and fluorescence. Where possible,

420 fluorescence data was primarily used to define cell boundaries (not available in the $C$.

421 elegans dataset acquired online). In addition to a blank channel to store manual labels, all 
422 processed phase and fluorescence images were then automatically loaded as layers into

423 an Adobe Photoshop document. We used 4-6 unique colors and the Pencil tool (for pixel-

424 level accuracy and no blending) to manually define object masks. Due to the 4-color

425 theorem ${ }^{54}$, this limited palette was sufficient to clearly distinguish individual object

426 instances from each other during annotation. This color simplification is not found in any

427 segmentation GUI, and it enabled faster manual annotation by reducing the need to select

428 new colors. It also eliminated the confusion caused by the use of similar but distinct

429 colors in adjacent regions, which we suspect is the principal cause for the misplaced

430 mask pixels that we observed in other datasets (e.g., cyto2).

431 The cell label layer was then exported as a PNG from Photoshop, read back into

432 MATLAB, and converted from the repeating N-color labels to a standard 16-bit integer

433 label matrix, where each object is assigned a unique integer from 1 to the number of cells

434 (background is 0). Because integer labels cannot be interpolated, we then performed a

435 non-rigid image registration of the brightfield channel to the binary label mask to achieve

436 better brightfield correlation to ground truth masks. All images in our ground-truth

437 dataset have been registered in this manner.

439 Choosing Segmentation algorithms

440 Three main factors contributed to the choice of algorithms highlighted in this

441 study: $(i)$ specificity to bacterial phase contrast images, (ii) success and community

442 adoption, especially for bioimage segmentation, and (iii) feasibility of installation,

443 training, and use. SuperSegger, Morphometrics, and MiSiC were selected because they

444 specifically targeted the problem of bacterial phase contrast segmentation ${ }^{15,23,55}$. Packages 
445 such as BactMAP, BacStalk, Cellprofiler, CellShape, ColiCoords, Cytokit,

446 MicroAnalyzer, MicrobeJ, Oufti, and Schnitzcells incorporate limited novel segmentation

447 solutions and instead aim to provide tools for single-cell analysis such as lineage tracing

448 and protein tracking $8,9,14,18-20,25,56-58$. Furthermore, the segmentation that these programs

449 perform depends broadly on thresholding and watershed techniques; therefore,

450 Morphometrics is a reasonable proxy for their segmentation capabilities. We were unable

451 to locate code or training data for $\mathrm{BASCA}^{11}$. Ilastik is a popular interactive machine-

452 learning tool for bioimage segmentation, but training it using a manual interface was not

453 feasible on a large and diverse dataset such as our own ${ }^{21}$. Among DNN approaches, Mask

454 R-CNN was selected because it is a popular architecture for handling typical image

455 segmentation tasks. It was also used in the segmentation and tracking package Usiigaci ${ }^{24}$.

456 U-Net architectures have been implemented in a number of algorithms, including

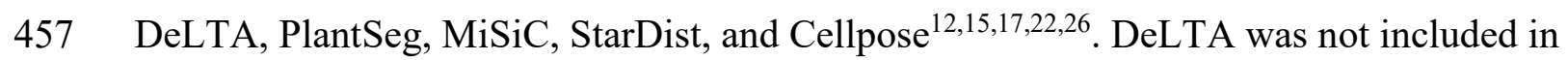

458 this study because it operates similarly to MiSiC and was designed specifically for

459 mother machine microfluidics analysis. DeLTA 2.0 was recently released to additionally

460 segment confluent cell growth on agarose pads, but it remains quite similar to MiSiC in

461 implementation ${ }^{59}$. PlantSeg could, in principle, be trained on bacterial micrographs, but

462 we determined that its edge-focused design meant to segment bright plant cell wall

463 features would not offer any advancements over the remaining U-Net methods that we

464 tested.

465

466 Training and tuning segmentation algorithms 
468 a given dataset. These include pre-processing such as image rescaling (often to put cells

469 into a particular pixel diameter range), contrast adjustment, smoothing, and noise

470 addition. Morphometrics and SuperSegger were manually tuned to give the best results

471 on our benchmarking dataset. The neural network component of SuperSegger was not

472 retrained on our data, as this is a heavily manual process involving toggling watershed

473 lines on numerous segmentation examples. DNN-based algorithms are automatically

474 trained using our dataset, and the scripts we used to do so are available in our GitHub

475 repository. We adapted our data for MiSiC by transforming our instance labels into

476 interior and boundary masks. Training documentation for MiSiC is not published.

477 Training and evaluation parameters for MiSiC were tuned according to correspondence

478 with the MiSiC authors. Cellpose and StarDist were trained with the default parameters

479 provided in their documentation. StarDist has an additional tool to optimize image pre-

480 processing parameters on our dataset, which we utilized.

\section{Evaluating segmentation algorithms}

All algorithms were evaluated on our benchmarking dataset with manually or

484 automatically optimized parameters. We provide both the raw segmentation results for all

485 test images by each tested algorithm as well as the models and model-training scripts

486 required to reproduce our results. Before evaluating IoU or JI, small masks at image

487 boundaries were removed for both the ground-truth and predicted masks. IoU and JI are

488 calculated on a per-image basis and, where shown, are averaged with equal weighting

489 over the image set or field of view. 
491 first measuring the fraction of each predicted cell that overlaps with each ground truth

492 cell. A predicted cell is assigned to a ground-truth cell if the overlap ratio is $\geq 0.75$,

493 meaning that at least three quarters of the predicted cell lies within the ground-truth cell.

494 If several predicted cells are matched to a ground-truth cell, the number of surplus

495 matches is taken to be the number of segmentation errors. If no cells are matched to a

496 ground-truth cell, then the error is taken to be 1.

498 Leveraging Omnipose to accelerate manual annotation

499 Omnipose was periodically trained on our growing dataset to make initial cell

500 labels. These were converted into an N-color representation and loaded into Photoshop

501 for manual correction. A subset of our cytosol GFP channels were sufficient for training

502 Omnipose to segment based on fluorescence, and the resulting trained model enabled

503 higher-quality initial cell labels for GFP-expressing samples than could be achieved from

504 intermediate phase contrast models (e.g., V. cholerae).

505

506 Defining the Omnipose prediction classes

507 Omnipose predicts four classes: two flow components, the distance field, and a

508 boundary field. Our distance field is found by solving the eikonal equation

$$
|\vec{\nabla} \phi(\vec{x})|=\frac{1}{f(\vec{x})}
$$

510 where $f$ represents the speed at a point $\vec{x}$. The Godunov upwind discretization of the

511 eikonal equation is 


$$
\left(\frac{\max \left(\phi_{i, j}-\min \left(\phi_{i-1, j}, \phi_{i+1, j}\right), 0\right)}{\Delta x}\right)^{2}+\left(\frac{\max \left(\phi_{i, j}-\min \left(\phi_{1, j-1}, \phi_{i, j+1}\right), 0\right)}{\Delta y}\right)^{2}=\frac{1}{f_{i, j}}
$$

514 follows. Our key contribution to this algorithm is the addition of ordinal sampling to

515 boost both convergence and smoothness of the final distance field.

$516 \quad 2 D$ update function for $\phi_{i, j}$ on a cartesian grid

517 1. Find neighboring points for cardinal axes $(\Delta x=\Delta y=\delta)$ :

$$
\phi^{\min x}=\min \left(\phi_{i-1, j}, \phi_{i+1, j}\right), \quad \phi^{\operatorname{miny}}=\min \left(\phi_{i, j-1}, \phi_{i, j+1}\right)
$$

2. Find neighboring points for ordinal axes $\left(\hat{x} \cdot \hat{a}=\hat{y} \cdot \hat{b}=\frac{\sqrt{2}}{2}, \frac{\Delta a}{\Delta x}=\frac{\Delta b}{\Delta y}=\sqrt{2} \delta\right)$ :

$$
\phi^{\text {mina }}=\min \left(\phi_{i-1, j-1}, \phi_{i+1, j+1}\right), \quad \phi^{\operatorname{minb}}=\min \left(\phi_{i+1, j-1}, \phi_{i-1, j+1}\right)
$$

3. Calculate update along cardinal axes:

$$
\text { if }\left|\phi^{\operatorname{minx}}-\phi^{\operatorname{miny}}\right|>\frac{\sqrt{2} \delta}{f_{i, j}}
$$

$$
U^{x y}=\min \left(\phi^{\min x}, \phi^{\text {miny }}\right)+\frac{\delta}{f_{i, j}}
$$

else:

4. Calculate update along ordinal axes:

$$
\text { if }\left|\phi^{\text {mina }}-\phi^{\operatorname{minb}}\right|>\frac{2 \delta}{f_{i, j}} \text { : }
$$

$$
U^{a b}=\min \left(\phi^{\operatorname{mina}}, \phi^{\operatorname{minb}}\right)+\frac{\sqrt{2} \delta}{f_{i, j}}
$$

else:

5. Update with geometric mean:

$$
U^{a b}=\frac{1}{2}\left(\phi^{\text {mina }}+\phi^{\text {minb }}+\sqrt{4\left(\frac{\delta}{f_{i, j}}\right)^{2}-\left(\phi^{\text {mina }}-\phi^{\operatorname{minb}}\right)^{2}}\right)
$$

$$
\phi_{i, j}=\sqrt{U^{x y} U^{a b}}
$$


$534 \delta=f_{i, j}$ to obtain the signed distance field used in Omnipose. The flow field components

535 are defined by the normalized gradient of this distance field $\phi$. The boundary field is

536 defined by points satisfying $0<\phi<1$. For network prediction, the boundary map is

537 converted to the logits (inverse sigmoid) representation, such that points in the range

$538[0,1]$ are mapped to $[-5,5]$. For consistent value ranges across prediction classes, the

539 flow components are multiplied by 5 and all background values of the distance field $(\phi=$

$540 \quad 0)$ are set to -5 .

542 Omnipose network architecture

543 The DNN used for Omnipose is a minor modification of that used in Cellpose: a

544 U-net architecture with two residual blocks per scale, each with two convolutional

545 layers ${ }^{12}$. Omnipose introduces a dropout layer before the densely connected layer ${ }^{60}$,

546 which we incorporated into the shared Cellpose and Omnipose architecture moving

547 forward. However, Cellpose models utilized in this study are trained without dropout.

549 Rescaling flow field by divergence

550 During training, the ground truth data is augmented by a random affine

551 transformation. The original implementation, and the one which yields the best results,

552 linearly interpolates the transformed field. This reduces the magnitude of the otherwise

553 normalized field in regions of divergence, i.e., at boundaries and skeletons. A

554 renormalized field (obtained either from the transformed field or as the normalized

555 gradient of the transformed heat distribution) often has artifacts at cell boundaries and 
556 skeletons, so the interpolated field effectively reduces the influence of these artifacts on

557 training. We reason that this feature explains the superior performance of interpolated

558 field training over renormalized fields, despite the latter being the nominal goal of the

559 algorithm.

560 Pixels at cell boundaries, however, consequently do not move far (less than 1px)

561 under Euler integration due to the low magnitude of the predicted field at cell boundaries.

562 Our solution in Omnipose is to rescale the flow field by the magnitude of the divergence.

563 The divergence is most positive at the cell boundaries (where pixels need to move) and

564 most negative at cell skeletons (where pixels need to stop). We therefore rescale the

565 divergence from 0 to 1 and multiply the normalized flow field by this new magnitude

566 map. This forces boundary pixels of neighboring cells to quickly diverge and allow for

567 accurate pixel clustering to obtain the final segmentation.

568

569 Novel diameter metric

570 The size models of Cellpose are trained to estimate the average cell 'diameter',

571 taken to be the diameter of the circle of equivalent area:

$$
d=2 \mathrm{R}=2 \sqrt{\frac{A}{\pi}}
$$

This metric as a basis for rescaling is problematic when cells are growing in

574 length but not width (Extended Data Fig. 7). Log-phase bacterial cell area grows

575 exponentially with time, and so too does the scale factor, eventually resulting in a

576 rescaled image that is too small for Cellpose to segment. 
578 bacteria, as the width - and therefore the distance to the closest boundary - remains

579 constant. To define a formula consistent with the previous definition in the case of a

580 circular cell, we consider mean of the distance field over the cell:

$$
\bar{\phi}=\frac{1}{\pi R^{2}} \int_{0}^{2 \pi} \int_{0}^{R}(R-r) r d r d \theta=\frac{1}{\pi R^{2}}\left(\frac{\pi}{3} R^{3}\right)=\frac{R}{3}
$$

This allows us to define a new 'effective diameter' as

$$
d=2 R=6 \bar{\phi}
$$

Aside from agreeing with the previous scaling method $(*)$ for round

585 morphologies, this definition exhibits excellent consistency across time (Extended Data

586 Fig. 7). This consistency is also critical for training on datasets with wide distributions in

587 cell areas that require rescaling, such as the Cellpose datasets. Finally, the raw distance

588 field output of Omnipose can directly be used directly in (**) to estimate average cell

589 diameter, which is used in our code to automatically toggle on features that improve

590 mask reconstruction performance for small cells.

\section{Gamma augmentation}

To make the network robust against changes in exposure/contrast, the training

594 images are now raised to a random power (gamma) between 0.5 and 1.25 , simulating the

595 varying levels of contrast that are observed experimentally with different light sources,

596 objectives, and exposure times. 


\section{$600 \quad$ Alleviating class imbalance}

601 Class imbalance remains a challenge in many machine learning applications ${ }^{61}$. In

602 our dataset, foreground pixels (cells) take up anywhere from 1 to 75 percent of a given

603 training image, with the rest being background pixels that the network must only learn to

604 ignore (i.e., assign a constant output of -5 for distance and boundary logits). We

605 implemented several changes to the loss function to emphasize foreground objects,

606 including weighting by the distance field and averaging some loss terms only over

607 foreground pixels. Our training augmentation function also attempts many random crop

608 and resizing passes until a field of view with foreground pixels is selected (this may take

609 several attempts for sparse images, but adds very little time to training).

\section{Image normalization}

612 To manage different image exposure levels, Cellpose automatically rescales

613 images such that pixels in the 1 st percentile of intensity are set to 0 and those in the 99th

614 percentile are sent to 1 . This percentile rescaling is preferred over blind min-max

615 rescaling because bubbles or glass can cause small bright spots in the image. However,

616 we found that images containing single cells (low intensity) in a wide field of media

617 (high intensity) would become badly clipped due to the foreground-background class

618 imbalance. To solve this, we changed the percentile range from 0.01 to 99.99.

620 Data availability 
621 Ground truth images and labels generated for this study are hosted on the Cellpose

622 website (http://www.cellpose.org/dataset_omnipose) and listed on the Papers With Code

623 database (https://paperswithcode.com/dataset/bpcis).

624

625 Code availability

626 Python and MATLAB scripts generated for this study is available from GitHub at

$627 \mathrm{https} / /$ github.com/kevinjohncutler/omnipose. Omnipose is available as part of the

628 Cellpose package at https://github.com/mouseland/cellpose. 


\section{Acknowledgements}

630 The authors wish to thank members of the Mougous and Wiggins laboratories for helpful

631 suggestions, See-Yeun Ting for assistance with experiments, Teresa Lo for assistance

632 with image acquisition, Sophie Sichel and Nina Salama lab for growing, fixing, and

633 staining H. pylori samples for in-house imaging, and David Kysela, Maxime Jacq and

634 Yves Brun for providing C. crescentus images. This work was supported by the NIH

635 (AI080609 to JDM, GM128191 to PAW, T32GM008268 to KJC). JDM is an HHMI

636 Investigator.

\section{Competing interests}

639 The authors declare no competing interests.

\section{Author contributions}

642 KJC, PAW and JDM conceived the study. KJC performed experiments, analyzed data,

643 and wrote the code. KJC, PAW, and JDM wrote the manuscript. CS edited the

644 manuscript and assisted in code development.

\section{Author Information}

647 Correspondence and requests for materials should be addressed to J.D.M.

648 (mougous@u.washington.edu) or P.A.W. (pwiggins@uw.edu). 


\section{References}

6511 Jeckel, H. \& Drescher, K. Advances and opportunities in image analysis of

652

653 bacterial cells and communities. FEMS Microbiol Rev 45,

6542 Bali, A. \& Singh, S. N. in IEEE Xplore 113-120 (IEEE, 2015).

6553 Lucas, A. M. et al. Open-source deep-learning software for bioimage

656 segmentation. Mol Biol Cell 32, 823-829, doi:10.1091/mbc.E20-10-0660 (2021).

6574 Kysela, D. T., Randich, A. M., Caccamo, P. D. \& Brun, Y. V. Diversity Takes Shape: Understanding the Mechanistic and Adaptive Basis of Bacterial Morphology. PLoS Biol 14, e1002565, doi:10.1371/journal.pbio.1002565 (2016).

6605 Jones, S. E. \& Elliot, M. A. 'Exploring' the regulation of Streptomyces growth and development. Curr Opin Microbiol 42, 25-30, doi:10.1016/j.mib.2017.09.009 (2018).

6636 Caccamo, P. D. \& Brun, Y. V. The Molecular Basis of Noncanonical Bacterial

664 Morphology. Trends Microbiol 26, 191-208, doi:10.1016/j.tim.2017.09.012

665 (2018).

6667 Behera, B. et al. Emerging technologies for antibiotic susceptibility testing.

667 Biosens Bioelectron 142, 111552, doi:10.1016/j.bios.2019.111552 (2019).

6688 Paintdakhi, A. et al. Oufti: an integrated software package for high-accuracy, high-throughput quantitative microscopy analysis. Molecular microbiology 99 , 767-777, doi:10.1111/mmi.13264 (2016).

6719 Ducret, A., Quardokus, E. M. \& Brun, Y. V. MicrobeJ, a tool for high throughput bacterial cell detection and quantitative analysis. Nat Microbiol 1, 16077, doi:10.1038/nmicrobiol.2016.77 (2016).

67410 Tropini, C., Earle, K. A., Huang, K. C. \& Sonnenburg, J. L. The Gut Microbiome: Connecting Spatial Organization to Function. Cell Host Microbe 21, 433-442, doi:10.1016/j.chom.2017.03.010 (2017).

$67711 \quad$ Balomenos, A. D. et al. Image analysis driven single-cell analytics for systems 678 microbiology. BMC Syst Biol 11, 43, doi:10.1186/s12918-017-0399-z (2017).

67912 Stringer, C., Wang, T., Michaelos, M. \& Pachitariu, M. Cellpose: a generalist algorithm for cellular segmentation. Nature methods 18, 100-106, doi:10.1038/s41592-020-01018-x (2021).

68213 Stylianidou, S., Brennan, C., Nissen, S. B., Kuwada, N. J. \& Wiggins, P. A. 
bacterial cells. Molecular microbiology 102, 690-700, doi:10.1111/mmi.13486 (2016).

14 van Raaphorst, R., Kjos, M. \& Veening, J. W. BactMAP: An R package for integrating, analyzing and visualizing bacterial microscopy data. Molecular microbiology 113, 297-308, doi:10.1111/mmi.14417 (2020).

15 Panigrahi, S. et al. Misic, a general deep learning-based method for the highthroughput cell segmentation of complex bacterial communities. Elife 10, doi:10.7554/eLife.65151 (2021).

16 Bannon, D. et al. DeepCell Kiosk: scaling deep learning-enabled cellular image analysis with Kubernetes. Nature methods 18, 43-45, doi:10.1038/s41592-02001023-0 (2021).

17 Lugagne, J. B., Lin, H. \& Dunlop, M. J. DeLTA: Automated cell segmentation, tracking, and lineage reconstruction using deep learning. PLoS computational biology 16, e1007673, doi:10.1371/journal.pcbi.1007673 (2020).

18 Smit, J. H., Li, Y., Warszawik, E. M., Herrmann, A. \& Cordes, T. ColiCoords: A Python package for the analysis of bacterial fluorescence microscopy data. PLoS One 14, e0217524, doi:10.1371/journal.pone.0217524 (2019).

19 Czech, E., Aksoy, B. A., Aksoy, P. \& Hammerbacher, J. Cytokit: a single-cell analysis toolkit for high dimensional fluorescent microscopy imaging. $B M C$ Bioinformatics 20, 448, doi:10.1186/s12859-019-3055-3 (2019).

20 McQuin, C. et al. CellProfiler 3.0: Next-generation image processing for biology. PLoS Biol 16, e2005970, doi:10.1371/journal.pbio.2005970 (2018).

21 Berg, S. et al. ilastik: interactive machine learning for (bio)image analysis. Nature methods 16, 1226-1232, doi:10.1038/s41592-019-0582-9 (2019).

22 Wolny, A. et al. Accurate and versatile 3D segmentation of plant tissues at cellular resolution. Elife 9, doi:10.7554/eLife.57613 (2020).

23 Ursell, T. et al. Rapid, precise quantification of bacterial cellular dimensions across a genomic-scale knockout library. BMC Biol 15, 17, doi:10.1186/s12915017-0348-8 (2017).

24 Tsai, H. F., Gajda, J., Sloan, T. F. W., Rares, A. \& Shen, A. Usiigaci: Instanceaware cell tracking in stain-free phase contrast microscopy enabled by machine learning. SoftwareX 9, 230-237, doi:https://doi.org/10.1016/j.softx.2019.02.007 (2019).

25 Reiner, J., Azran, G. \& Hyams, G. MicroAnalyzer: A Python Tool for Automated Bacterial Analysis with Fluorescence Microscopy. arXiv, doi:https://arxiv.org/abs/2009.12684 (2020). 
72026 Schmidt, U. et al. Cell Detection with Star-Convex Polygons. (2018).

72127 He, K., Gkioxari, G., Dollar, P. \& Girshick, R. Mask R-CNN. arXiv, 722 doi:https://arxiv.org/abs/1703.06870 (2018).

72328 Shal, K. \& Choudhry, M. S. Evolution of Deep Learning Algorithms for MRI-

724

725

726

29 Bean, G. J. et al. A22 disrupts the bacterial actin cytoskeleton by directly binding Based Brain Tumor Image Segmentation. Crit Rev Biomed Eng 49, 77-94, doi:10.1615/CritRevBiomedEng.2021035557 (2021). and inducing a low-affinity state in MreB. Biochemistry 48, 4852-4857, doi:10.1021/bi900014d (2009). replication crisis in deep-learning-based bioimage analysis. Nature methods $\mathbf{1 8}$, 1136-1144, doi:10.1038/s41592-021-01284-3 (2021).

Taha, A. A. \& Hanbury, A. Metrics for evaluating 3D medical image segmentation: analysis, selection, and tool. BMC Med Imaging 15, 29, doi:10.1186/s12880-015-0068-x (2015). (2017). discovering clusters in large spatial databases with noise. doi:10.1.1.121.9220 (1996).

Sethian, J. A. \& Vladimirsky, A. Ordered upwind methods for static HamiltonJacobi equations. Proc Natl Acad Sci U S A 98, 11069-11074, doi:10.1073/pnas.201222998 (2001). Computing. arXiv, doi:http://arXiv:2106.15869v3 (2021).

Ting, S. Y. et al. Bifunctional Immunity Proteins Protect Bacteria against FtsZTargeting ADP-Ribosylating Toxins. Cell 175, 1380-1392, doi:10.1016/j.cell.2018.09.037 (2018). 
39 Javer, A. et al. An open-source platform for analyzing and sharing worm-behavior data. Nature methods 15, 645-646, doi:10.1038/s41592-018-0112-1 (2018).

40 Ljosa, V., Sokolnicki, K. L. \& Carpenter, A. E. Annotated high-throughput microscopy image sets for validation. Nature methods $\mathbf{9}, 637$, doi:10.1038/nmeth.2083 (2012).

41 Cusick, J. A., Wellman, C. L. \& Demas, G. E. The call of the wild: using nonmodel systems to investigate microbiome-behaviour relationships. J Exp Biol 224, doi:10.1242/jeb.224485 (2021).

42 Wang, Z. Cell Segmentation for Image Cytometry: Advances, Insufficiencies, and Challenges. Cytometry A 95, 708-711, doi:10.1002/cyto.a.23686 (2019).

43 He, C. et al. Genome-resolved metagenomics reveals site-specific diversity of episymbiotic CPR bacteria and DPANN archaea in groundwater ecosystems. Nat Microbiol 6, 354-365, doi:10.1038/s41564-020-00840-5 (2021).

44 Looi, S. rotated_maskrcnn, < $\underline{\text { https://github.com/mrlooi/rotated maskrcnn }>}$ (2019).

45 Bharti, R. \& Grimm, D. G. Current challenges and best-practice protocols for microbiome analysis. Brief Bioinform 22, 178-193, doi:10.1093/bib/bbz155 (2021).

46 Smits, W. K., Kuipers, O. P. \& Veening, J. W. Phenotypic variation in bacteria: the role of feedback regulation. Nat Rev Microbiol 4, 259-271, doi:10.1038/nrmicro1381 (2006).

47 Bailey, J. et al. Essential gene deletions producing gigantic bacteria. PLoS genetics 15, e1008195, doi:10.1371/journal.pgen.1008195 (2019).

48 Cass, J. A., Stylianidou, S., Kuwada, N. J., Traxler, B. \& Wiggins, P. A. Probing bacterial cell biology using image cytometry. Molecular microbiology 103, 818828, doi:10.1111/mmi.13591 (2017).

49 Meberg, B. M., Sailer, F. C., Nelson, D. E. \& Young, K. D. Reconstruction of Escherichia coli mrcA (PBP 1a) mutants lacking multiple combinations of penicillin binding proteins. J Bacteriol 183, 6148-6149, doi:10.1128/JB.183.20.6148-6149.2001 (2001).

50 Lowenthal, A. C. et al. Functional analysis of the Helicobacter pylori flagellar switch proteins. J Bacteriol 191, 7147-7156, doi:10.1128/JB.00749-09 (2009).

51 Taylor, J. A. et al. Distinct cytoskeletal proteins define zones of enhanced cell wall synthesis in Helicobacter pylori. Elife 9, doi: 10.7554/eLife.52482 (2020). 
78952 Evinger, M. \& Agabian, N. Envelope-associated nucleoid from Caulobacter $790 \quad$ crescentus stalked and swarmer cells. J Bacteriol 132, 294-301,

791 doi:10.1128/jb.132.1.294-301.1977 (1977).

79253 Caccamo, P. D., Jacq, M., VanNieuwenhze, M. S. \& Brun, Y. V. A Division of

793

794

795

54 Robertson, N., Sanders, D. P., Seymour, P. \& Thomas, R. A new proof of the four-colour theorem. Electron. Res. Announc. Amer. Math. Soc., doi:https://doi.org/10.1090/S1079-6762-96-00003-0 (1996). Labor in the Recruitment and Topological Organization of a Bacterial Morphogenic Complex. Curr Biol 30, 3908-3922 e3904, doi:10.1016/j.cub.2020.07.063 (2020). SuperSegger: robust image segmentation, analysis and lineage tracking of bacterial cells. Molecular microbiology 102, 690-700, doi:10.1111/mmi.13486 (2016).

56 Hartmann, R., van Teeseling, M. C. F., Thanbichler, M. \& Drescher, K. BacStalk: A comprehensive and interactive image analysis software tool for bacterial cell biology. Molecular microbiology 114, 140-150, doi:10.1111/mmi.14501 (2020).

Goni-Moreno, A., Kim, J. \& de Lorenzo, V. CellShape: A user-friendly image analysis tool for quantitative visualization of bacterial cell factories inside. Biotechnol J 12, doi:10.1002/biot.201600323 (2017).

Young, J. W. et al. Measuring single-cell gene expression dynamics in bacteria using fluorescence time-lapse microscopy. Nature protocols $\mathbf{7}, 80-88$, doi:10.1038/nprot.2011.432 (2011). dynamics. bioRxiv, doi:https://doi.org/10.1101/2021.08.10.455795 (2021).

82162 Meberg, B. M., Sailer, F. C., Nelson, D. E. \& Young, K. D. Reconstruction of Dropout: a simple way to prevent neural networks from overfitting. The Journal of Machine Learning Research 15, 1929-1958 (2014). 
82563 Barbe, V. et al. Unique features revealed by the genome sequence of

826 Acinetobacter sp. ADP1, a versatile and naturally transformation competent bacterium. Nucleic Acids Res 32, 5766-5779, doi:10.1093/nar/gkh910 (2004).

$82864 \mathrm{Yu}, \mathrm{Y}$. et al. Genomic patterns of pathogen evolution revealed by comparison of

829 Burkholderia pseudomallei, the causative agent of melioidosis, to avirulent

$830 \quad$ Burkholderia thailandensis. BMC microbiology 6, 46 (2006).

83165 Allue-Guardia, A., Echazarreta, M., Koenig, S. S. K., Klose, K. E. \& Eppinger, 832 M. Closed Genome Sequence of Vibrio cholerae O1 El Tor Inaba Strain A1552.

833 Genome Announc 6, doi:10.1128/genomeA.00098-18 (2018).

83466 Stover, C. K. et al. Complete genome sequence of Pseudomonas aeruginosa 835 PA01, an opportunistic pathogen. Nature 406, 959-964 (2000). 

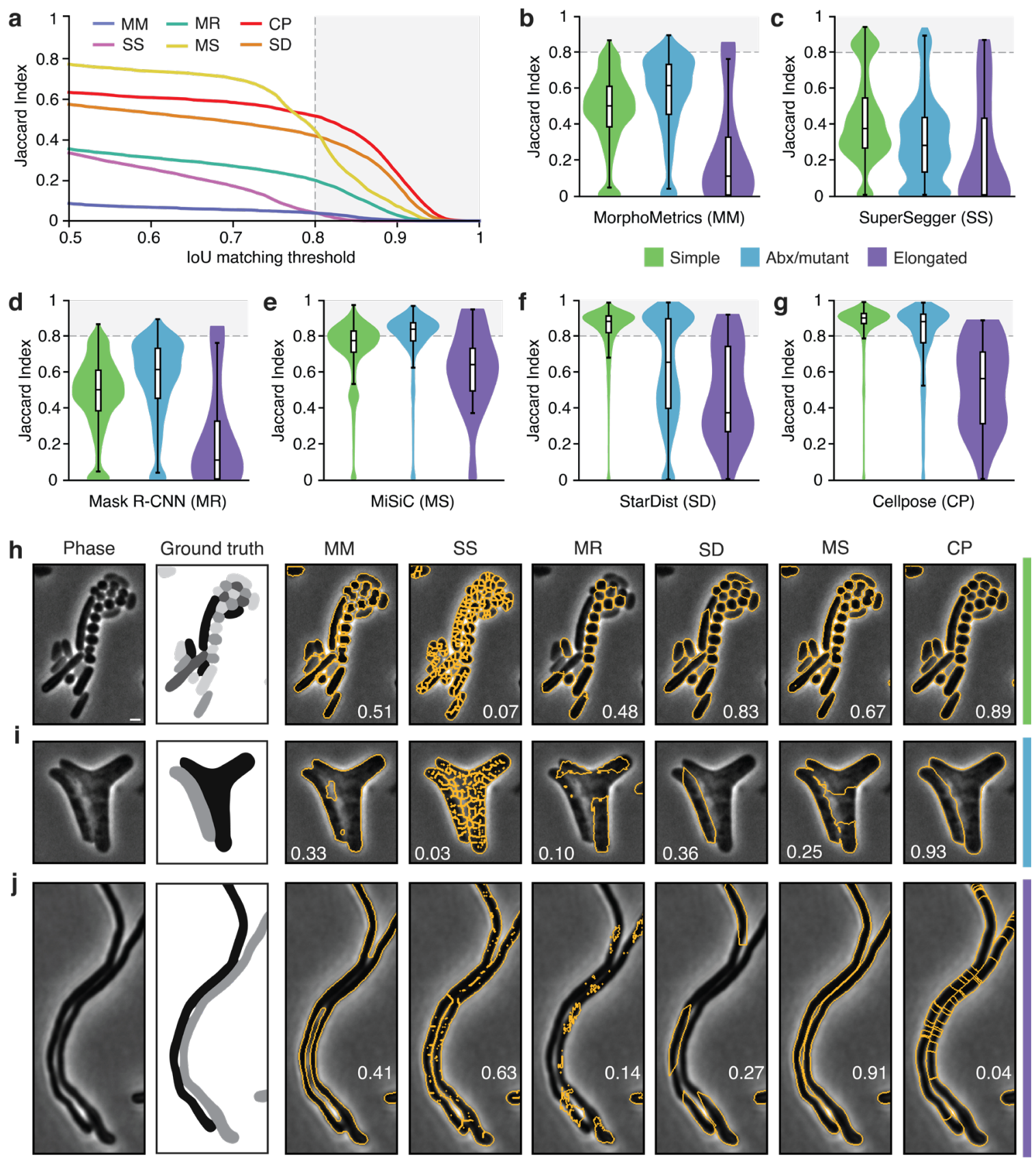

$\overline{\text { Traditional }}$
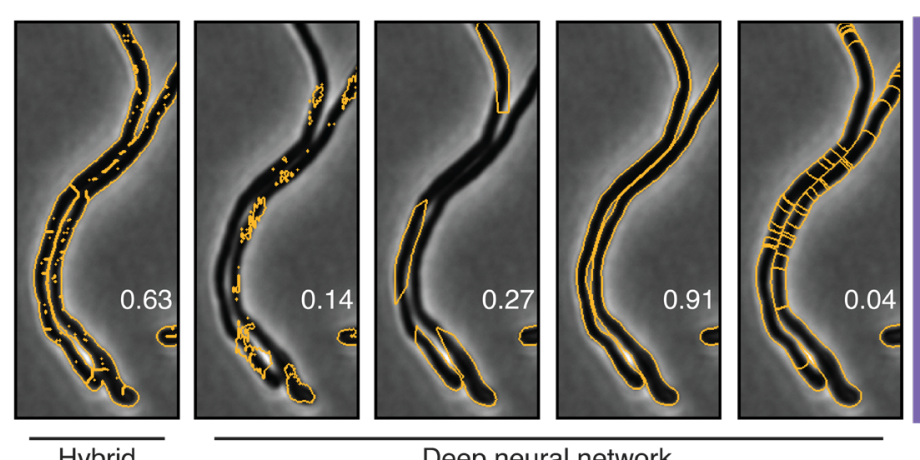

Deep neural network

Fig. 1 | Quantitative comparison of segmentation methods distinguishes Cellpose as a high performing algorithm. (a-g) Comparison of segmentation algorithm performance on our test dataset. (a) Overall performance measured by Jaccard Index (JI). The JI was calculated at the image level and values averaged across the dataset are displayed. Algorithm abbreviations defined in B-G. (b-g) Algorithm performance partitioned by cell type (Simple, $n=12,869 ;$ Abx/mutant, $n=6,138$; Elongated, $n=46$ ). Images were sorted into types as defined in Supplemental Table 1 (Abx, antibiotic). (h-j) Representative micrographs of cell type partitions analyzed in B-G, indicated by vertical bars at right. Ground-truth masks and predicted mask outlines generated by the indicated algorithm are displayed. Mean matched IoU values for cells shown are displayed within each micrograph. Bacteria displayed are (H) Vibrio cholerae, Pseudomonas aeruginosa, Bacillus subtilis, Staphylococcus aureus, (I) aztreonam-treated Escherichia coli CS703-1, and (J) Streptomyces pristinaespiralis. All images scaled equivalently; scale bar is $1 \mathrm{~mm}$. 
a

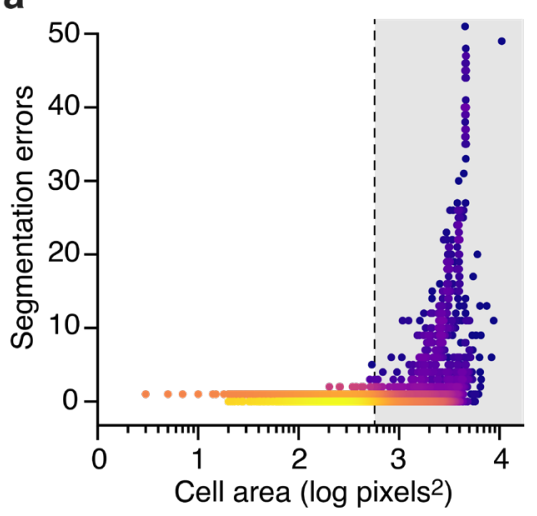

b
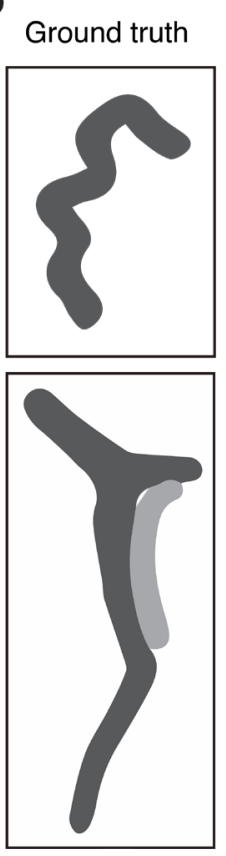
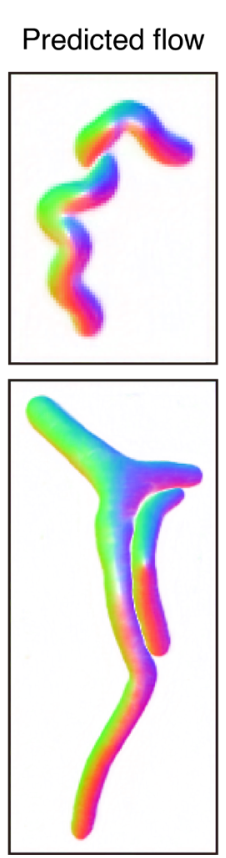

C

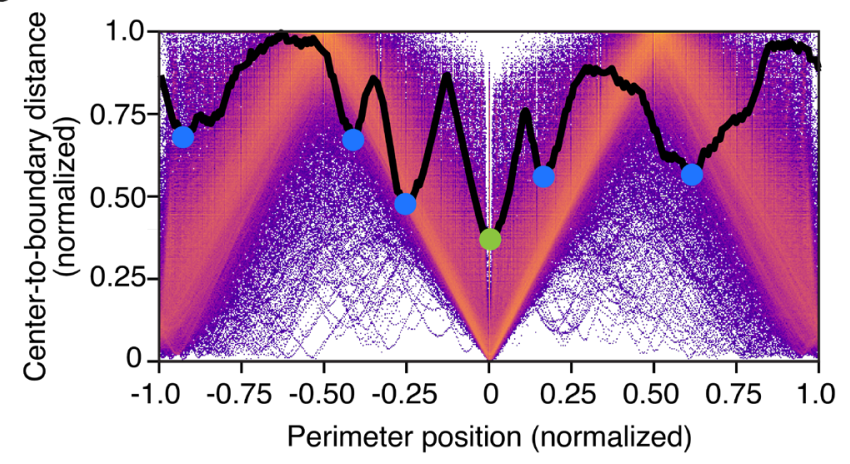

d

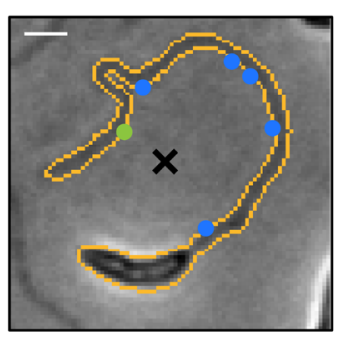

e

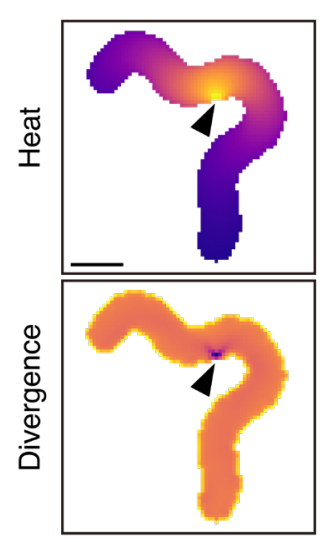

Fig. 2 | Cellpose over-segments extended, anisotropic cells. (a) Single-cell analysis of segmentation error as a function of cell area. Color represents density on a log scale. Gray box represents the top quartile of cell areas. (b) Representative examples exhibiting problematic flow fields. Corresponding boundary pixel trajectories are shown in black and final pixel locations in red. Predicted mask overlays are shown with mean matched IoU values. (c) Analysis of stochastic center-to-boundary distances. Distance from the center (median pixel coordinate) to each boundary pixel is normalized to a maximum of 1 . Position along the boundary is normalized from -1 to 1 and centered on the point closest to the median pixel. Center-toboundary for the cell in panel D is highlighted in black. (d) Representative cell with median coordinate outside the cell body (black X). Cellpose projects this point to the global minima of this function (green dot), but several other local minima exist (blue dots). (e) The heat distribution resulting from a projected cell center (black arrow). The normalized gradient corresponds to the divergence shown. Bacteria displayed are (a,e) Helicobacter pylori, (b) Escherichia coli CS703-1, both treated with aztreonam, and (d) Caulobacter crescentus grown in HIGG media. Scale bars are $1 \mu \mathrm{m}$. 
a
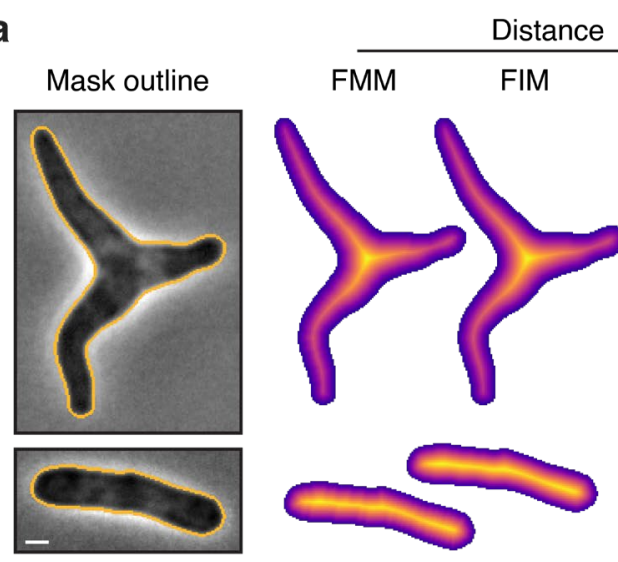

Difference

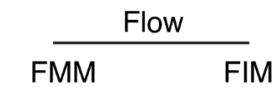

b Pixel trajectories

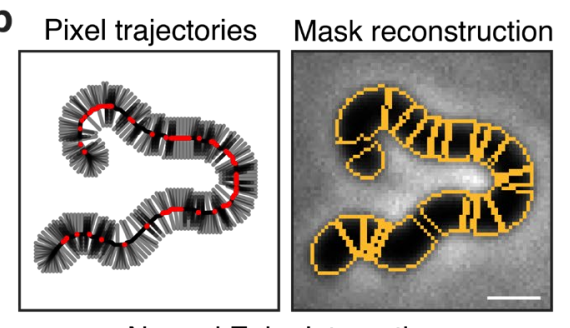

Normal Euler integration

Normal Euler integration

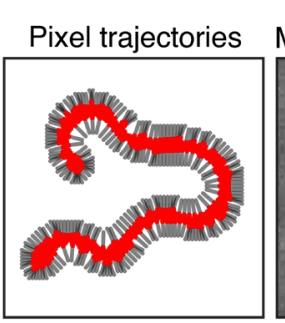

Mask reconstruction

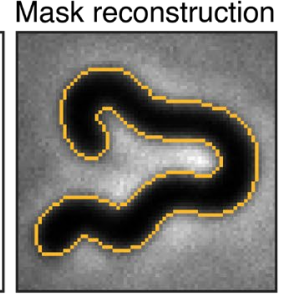

Suppressed Euler integration

Fig. 3 | Core innovations of Omnipose. (a) Comparison of distance field algorithms and corresponding flow fields. Fast Marching Method (FMM) produces ridges in the distance field resulting from pixelation on the cell mask boundary. Our smooth FIM algorithm minimizes these features. The difference image (FIM - FMM) highlights artifacts in the FMM method. Flow fields are calculated as the normalized gradient of the distance field. Boundary pixelation affects the FMM flow field deep into the cell, regardless of cell size. (b) Comparison of mask reconstruction algorithms on a smooth flow field (not shown). Left: boundary pixel trajectories and resulting mask outlines from standard Euler integration. Right: Trajectories and mask outlines under suppressed Euler integration. Red dots indicate the final positions of all cell pixels, not only the boundary pixels for which trajectories are displayed. Bacteria displayed are (a) Escherichia coli CS703-1 and (b) and Helicobacter pylori, both treated with aztreonam. Scale bars are $1 \mu \mathrm{m}$. 
a

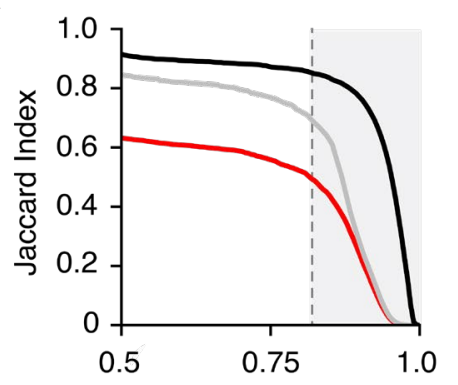

IoU matching threshold

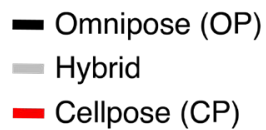

b

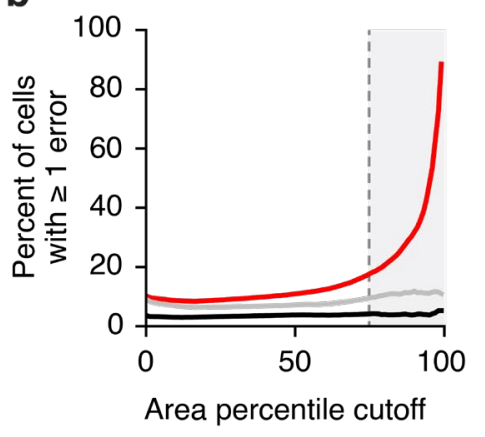

C

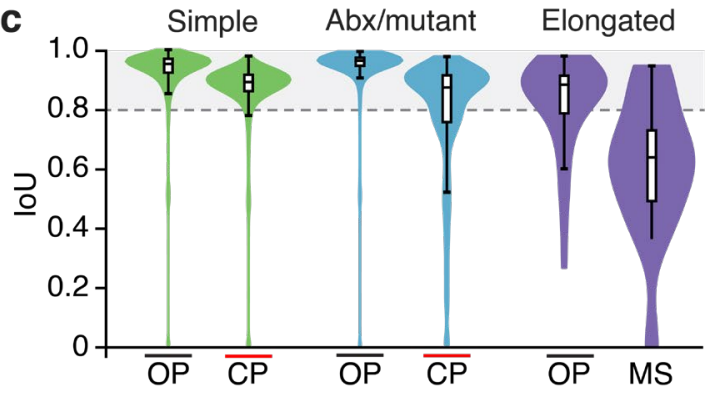

d
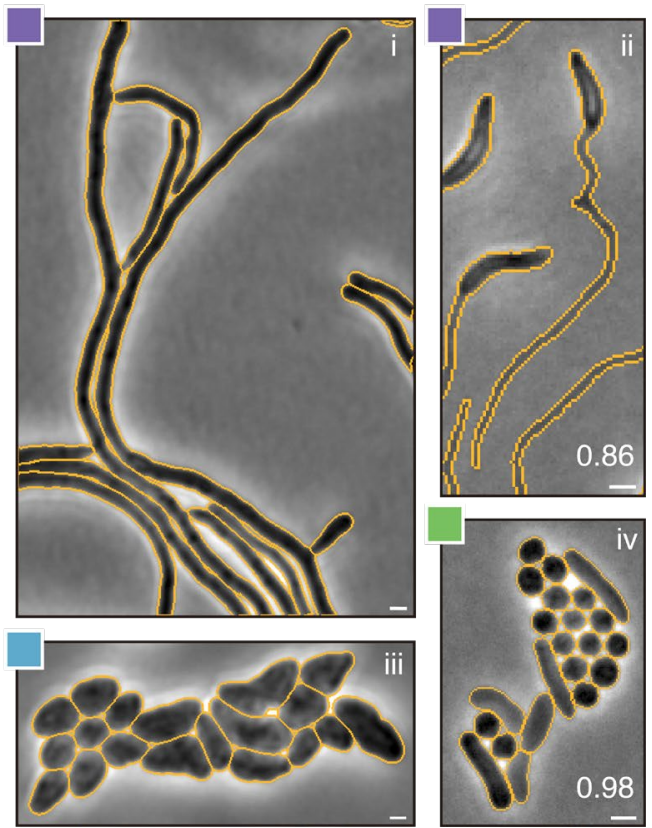

Fig. 4 | Omnipose outperforms Cellpose. (a) Overall performance measured by Jaccard Index (JI). The hybrid method (gray) is a variant of Cellpose that uses the original center-seeking flow output and the mask reconstruction of Omnipose. Gray box represents IoU $\geq 0.8$. (b) Quantification of segmentation performance by cell size. The percent of cells with at least one segmentation error is computed for cells in each area percentile group from 1 to 100. Gray box represents the top quartile. (c) Omnipose IoU distribution on our dataset compared to the next highest performing algorithm in each of three cell categories. (d) Example micrographs and Omnipose segmentation. Mean matched IoU values shown. Bacteria displayed are (i) Streptomyces pristinaespiralis, (ii) Caulobacter crescentus grown in HIGG media, (iii) Shigella flexneri treated with A22, (iv) mix of Pseudomonas aeruginosa, Staphylococcus aureus, Vibrio cholerae, and Bacillus subtilis. Scale bars are $1 \mu \mathrm{m}$. 


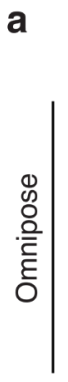

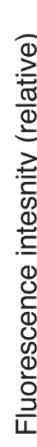

- S. protea tre1 $10^{1}$. protea tre $1 E 415 \mathrm{Q}$

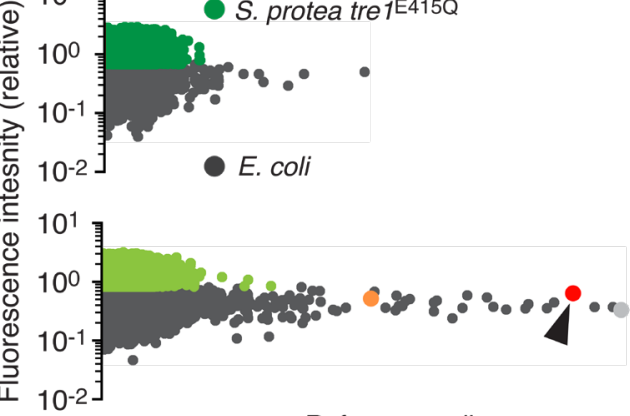

Reference cells

d
$\frac{0}{20}$
$\frac{00}{\Sigma}$

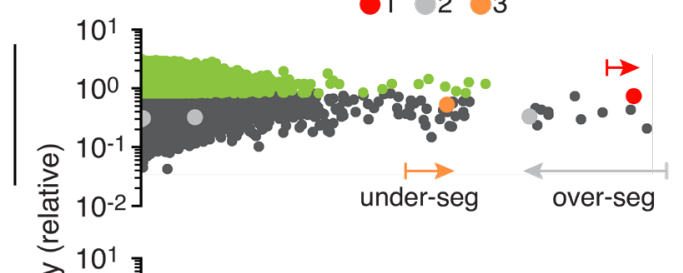

\begin{tabular}{l|}
0 \\
0 \\
0 \\
$\overline{\overline{0}}$ \\
\hline \\
0
\end{tabular}

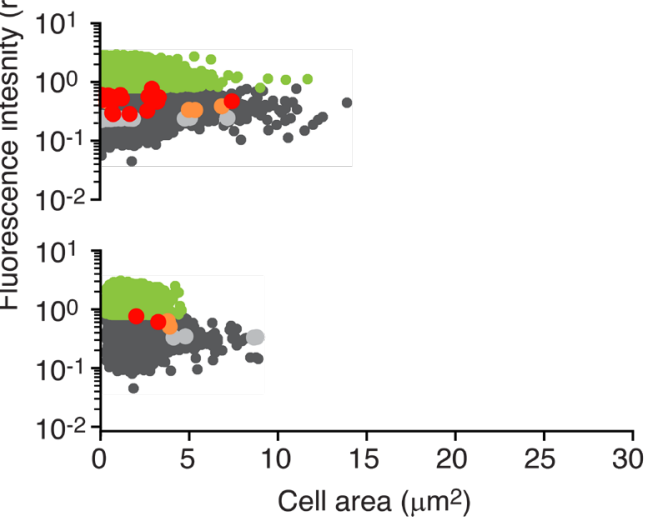

b

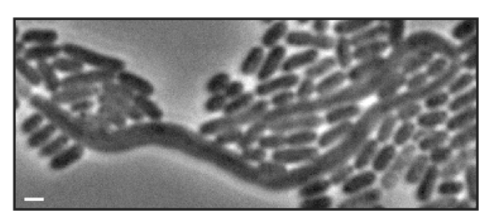

c

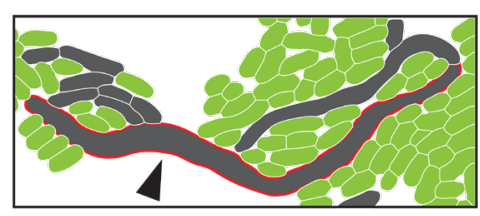

e
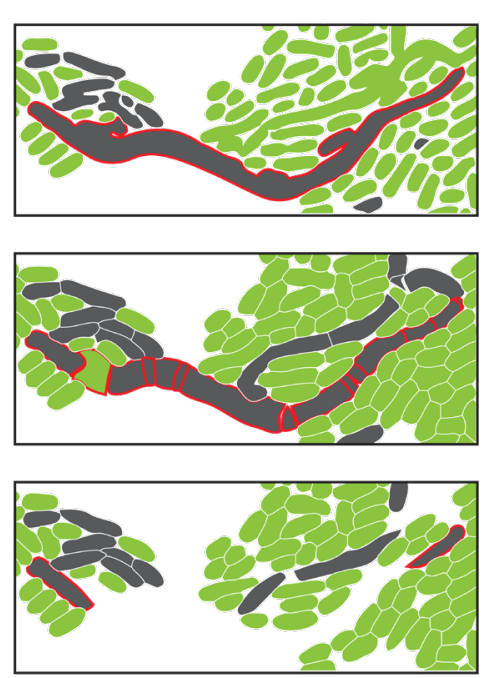

h

Omnipose (Bacteria and C. elegans model)

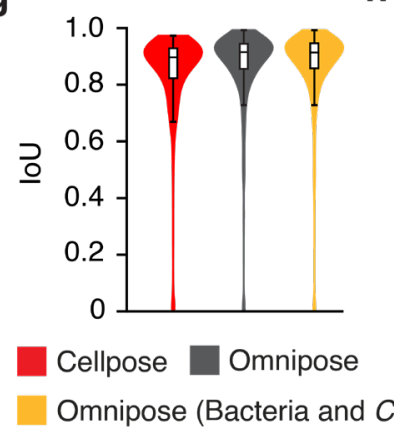

g

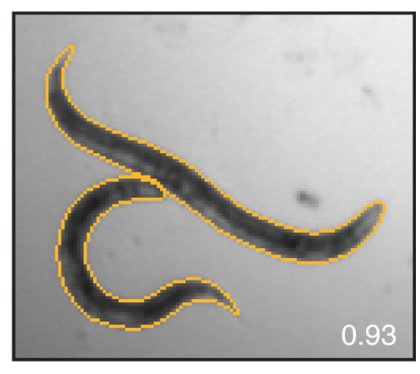

0.93 f

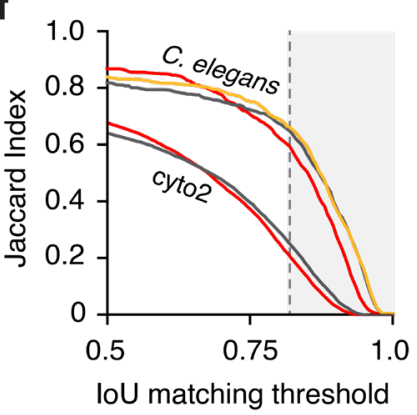

Fig. 5 | Omnipose can be applied to the study of bacterial and non-bacterial systems. (a)

Fluorescence/area population profile according to Omnipose segmentation in control and experimental conditions. K-means clustering on GFP fluorescence distinguishes $S$. proteamaculans tre 1/tre ${ }^{E 415 Q}$ (light/dark green markers) from E. coli (gray markers). (b) Example of extreme filamentation of E. coli in response to active Tre1. (c) Omnipose accurately segments all cells in the image. Largest cell indicated with black arrow. (d) MiSiC predicts large cell masks over both species. Cellpose and StarDist fail to predict any cells above $15 \mu \mathrm{m}^{2}$. (e) Example segmentation results highlighting typical errors encountered with MiSiC (under-segmentation), Cellpose (over-segmentation), and StarDist (incomplete masks). Mask mergers cause some E. coli to be misclassified as $S$. proteamaculans. Scale bar is $1 \mu \mathrm{m}$. (f) Performance of Omnipose and Cellpose on cyto2 and C. elegans datasets. Results for Omnipose trained on C. elegans (grey) or C. elegans and bacterial data (yellow) are shown. (g) IoU distribution for the masks predicted by each method on our C.elegans dataset. (h) Example segmentation of C. elegans in the BBBC010 dataset. 

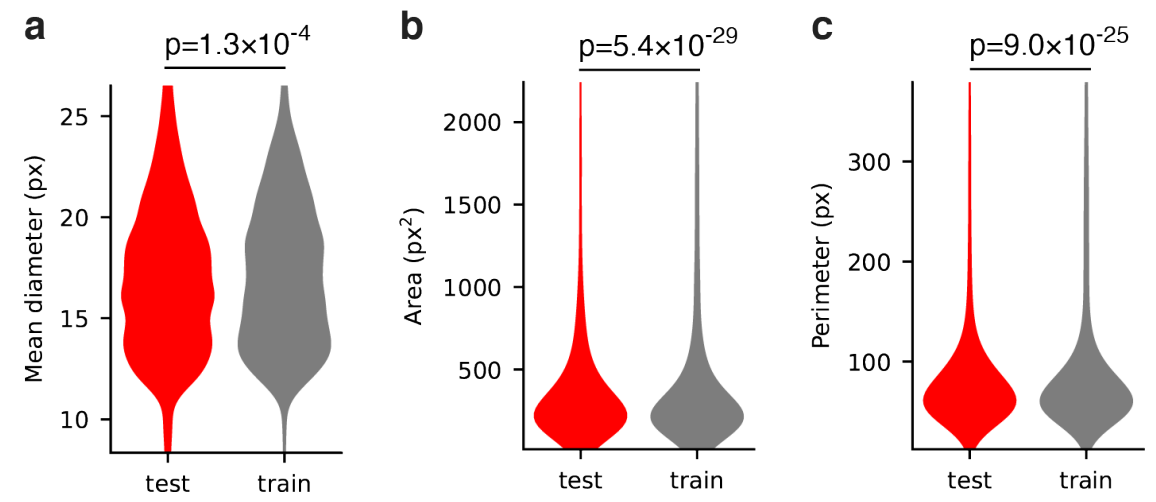

Extended Data Fig. 1 | Test dataset is representative of the training dataset. (a) Mean diameter, defined in Methods. (b) Cell area. (c) Cell perimeter. P-values are displayed for the two-sided KS test. 
a

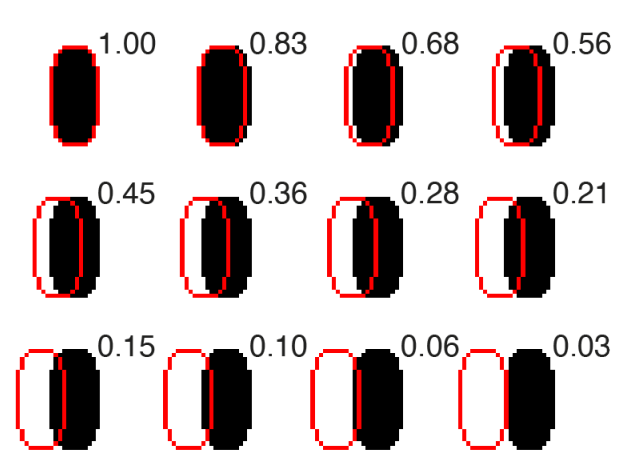

b

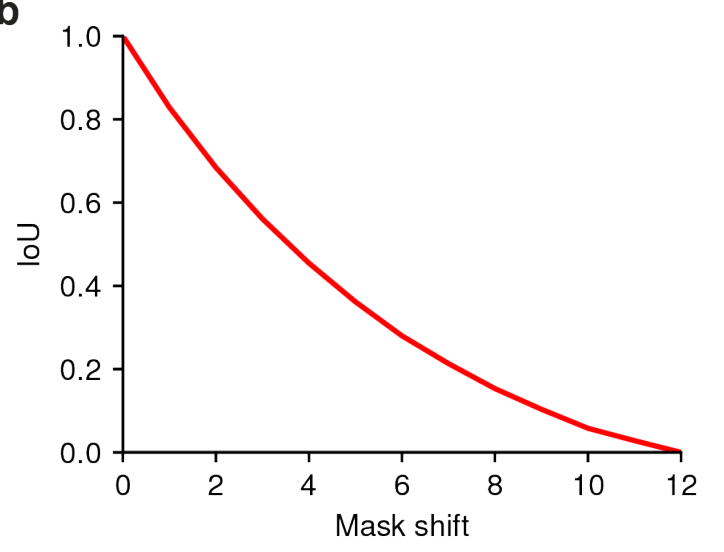

Extended Data Fig. 2 | IoU values for synthetic cell of typical size/resolution. (a) 0-12 pixel displacement of cell mask (red outline) and corresponding IoU values. (b) IoU decreases non-linearly for curved regions such as this synthetic cell. 


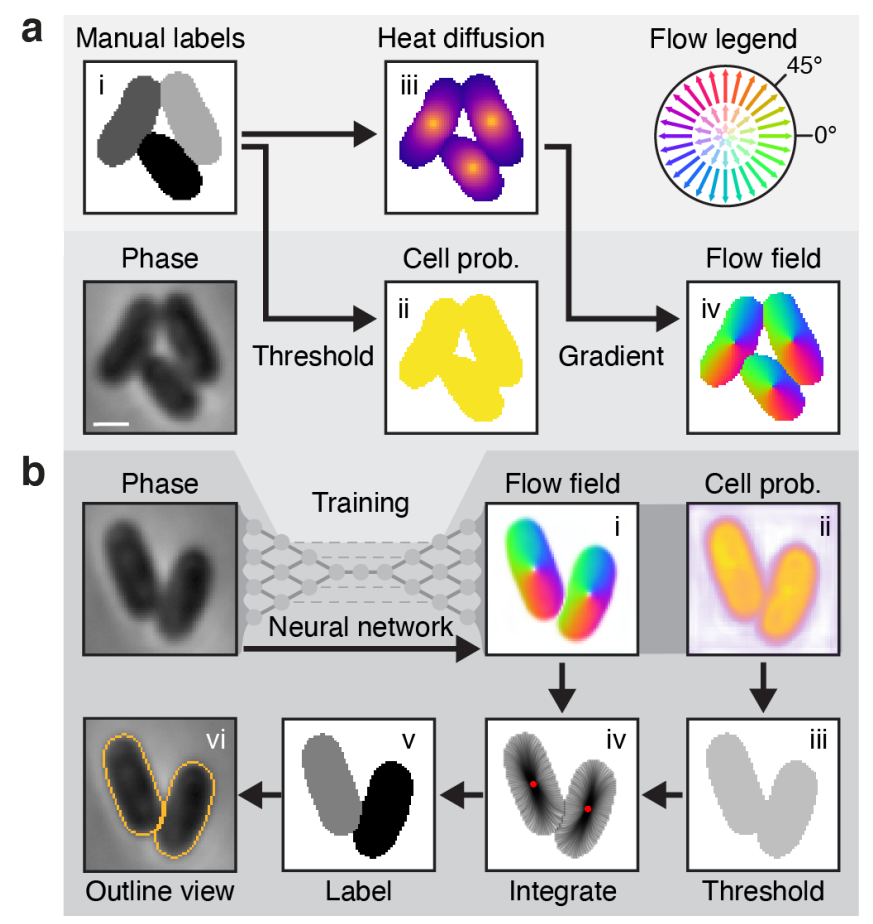

Extended Data Fig. 3 | Details of the Cellpose algorithm. (a) Stages of the Cellpose training pipeline. Ground truth masks $(i)$ are converted to cell probability (ii) by binary thresholding and a heat distribution (iii) by simulated diffusion from the median pixel coordinate. The flow field (iv) is defined by the normalized gradient of (iii). Color-magnitude representations of this vector field follow the flow legend diagram. The phase, cell probability, and flow fields are used to train the network. (b) Stages of the cellpose prediction pipeline. Phase images are processed by the trained cellpose network into the intermediate flow field and cell probability outputs $(i-i i)$. A binary threshold is applied to the probability to identify cell pixels (iii). Pixels are Euler-integrated under the flow field until they converge at common points. Boundary pixel trajectories are depicted in $i v$. Each pixel is assigned a unique label corresponding to the center to which it converged $(v)$. This segmentation result is commonly depicted in an outline view (vi). Bacteria shown are Escherichia coli. Scale bar is $1 \mu \mathrm{m}$. 
a

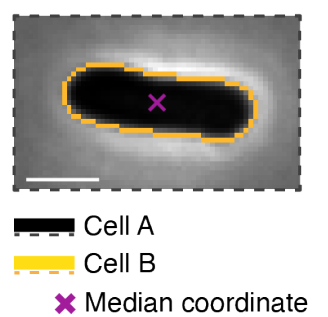

C

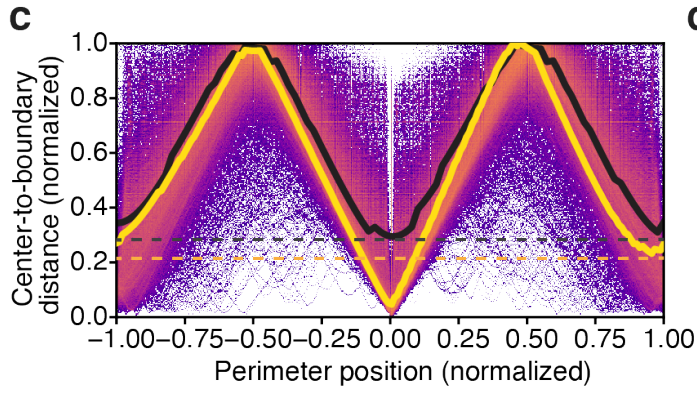

b

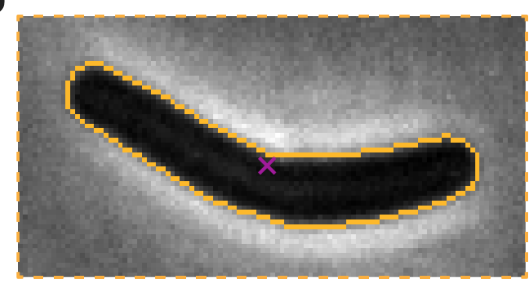

d

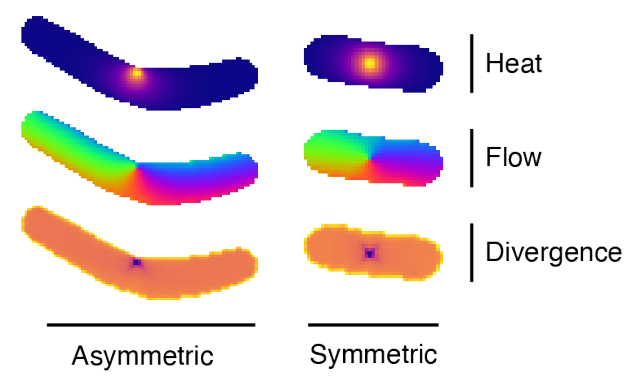

Extended Data Fig. 4 | Median coordinates are asymmetrically localized. (a) Center-to-boundary distance highlighted for two cells with non-projected median coordinates. Dashed lines indicate the larger of the two minima along the medial axis. (b) Rod-shaped E.coli with symmetric median coordinate. Symmetry of the center is reflected in A by equal high and low points corresponding to the extremal points along the long and short axes of the cell. (c) Curved B. subtilis with median coordinate asymmetrically close to the cell boundary. This asymmetry is reflected in A by a secondary minimum above the global minimum corresponding to the diametrically opposing point along the short axis of the cell. (d) These centers result in distinct flow fields reflecting the (a)symmetric of the cell center. Bacteria shown are (a) Escherichia coli and (b) Bacillus subtilis. Scale bar is $1 \mu \mathrm{m}$. Images scaled equivalently. 

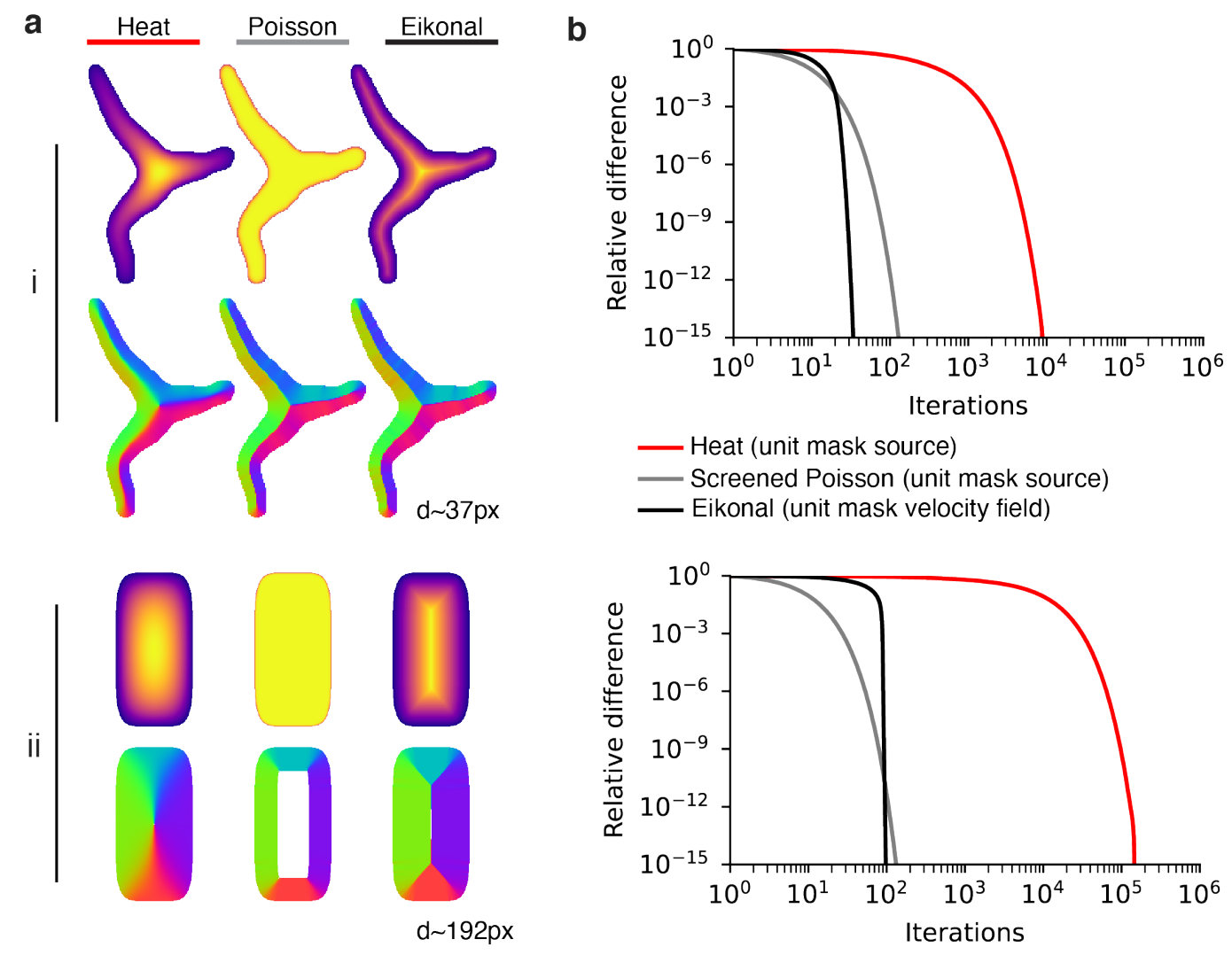

Extended Data Fig. 5 | Comparison of three algorithms for computing center-independent flow fields. Each field is defined by a partial differential equation with the mask at the source: time-independent heat equation, the screened Poisson equation, and the Eikonal equation. We solve these equations with iterative relaxation (see Methods). (a) Two example cells, the first drawn from our dataset with a mean diameter of $37 \mathrm{px}$ and a synthetic rod-shaped cell with a mean diameter of 192px. Cell $(i)$ exhibits heat-derived flow components pointing toward the skeleton near boundaries and toward the global cell center at the skeleton. Center-seeking flow components become problematic for mask reconstruction for more complicated cell geometries, namely those with oscillating thickness. The screened Poisson and Eikonal equations produce nearly identical flow fields (same direction, normalized magnitude). Cell (ii) reveals a core flaw in the screened Poisson solution: its derivative exceeds our available numerical precision, leading to a vanishing flow field at the center where the solution plateaus. Any cells of this size or larger will exhibit this issue. (b) Convergence measured by the average difference at each iteration (maximum normalized to 1 ) for cells $(i, i i)$. Our Eikonal solution converges faster than the other methods by a wide margin at typical cell diameters $(i)$. 


\section{a}
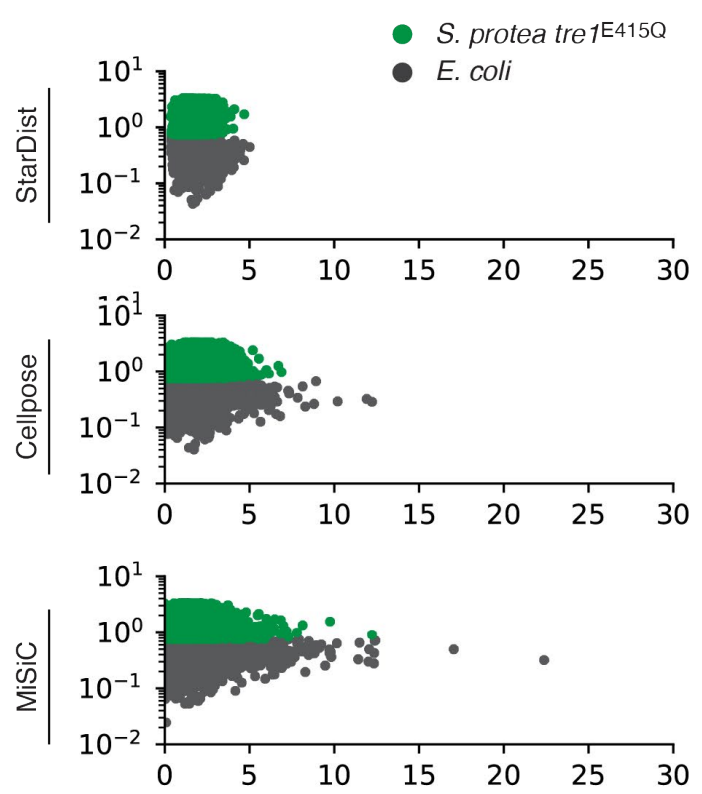

b
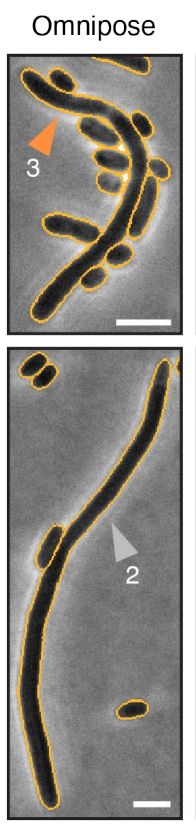

MiSiC
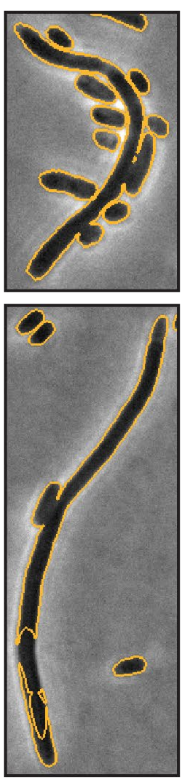

Cellpose
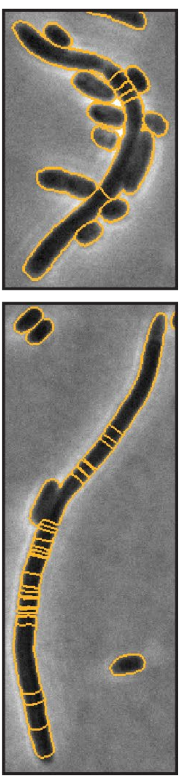

StarDist
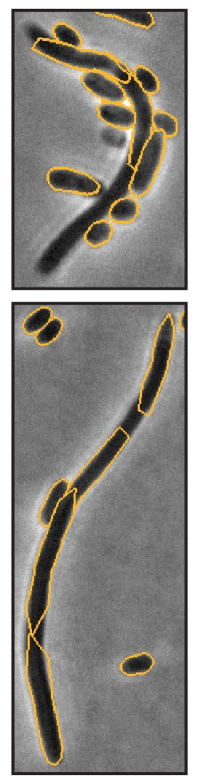

Extended Data Fig. 6 | Controls and additional examples. (a) Controls segmented by StarDist, Cellpose, and MiSiC. Notably, Cellpose and MiSiC exhibit an enrichment of larger cells even in the control, a consequence of both under-segmented (merged) cells as well as fragments of over-segmented large cells. (b) Cells 2 and 3 highlighted in orange and gray plotted in Fig. 5a,d. Scale bars are $1 \mu \mathrm{m}$. 
a

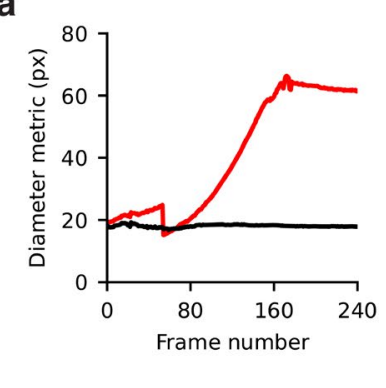

b

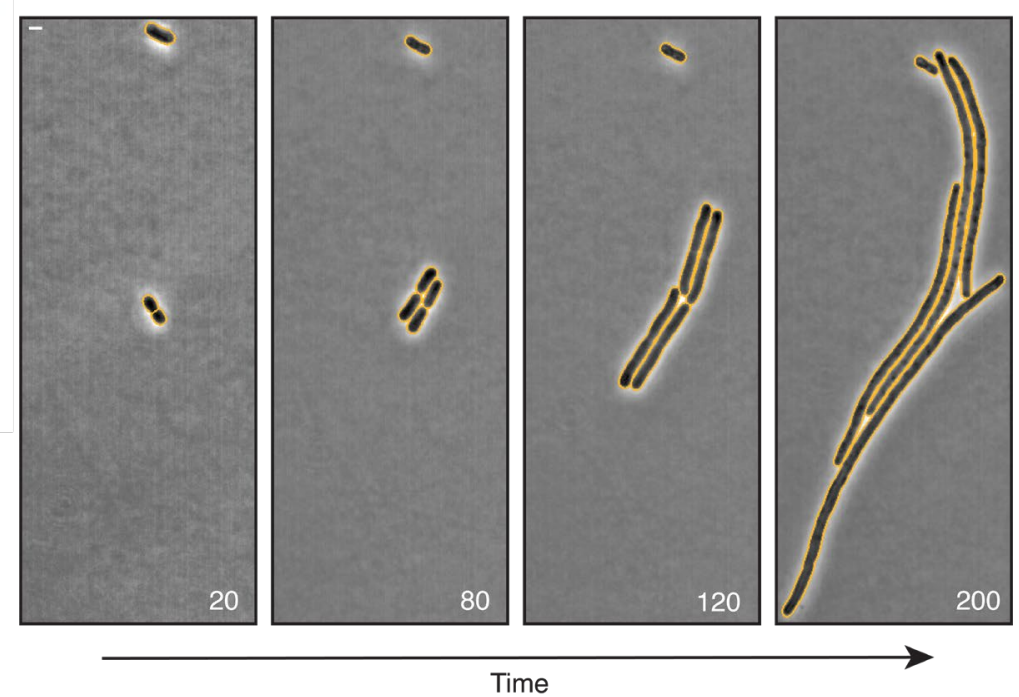

Extended Data Fig. $7 \mid$ Comparison of diameter metrics on a filamentous microcolony time lapse. (a). Cellpose diameter metric is the diameter of the circle with equivalent area. Omnipose diameter metric is proportional to the mean of the distance transform. (b) Bacteria displayed are A. baylyi transformed with a $\Delta f t s N:: k a n$ PCR fragment. Scale bar is $1 \mu \mathrm{m}$. 


\section{Extended Data Table 1.}

\begin{tabular}{|c|c|c|c|c|c|c|}
\hline Species & Strain & $\begin{array}{l}\text { Image } \\
\text { count }\end{array}$ & Cell Count & $\begin{array}{l}\text { Cells in } \\
\text { GT }\end{array}$ & $\begin{array}{l}\text { Percent } \\
\text { of GT }\end{array}$ & Notes \\
\hline \multirow[t]{4}{*}{ Escherichia coli } & \multirow[t]{3}{*}{ DH5 $\alpha$} & 1378 & 98200 & 9733 & 20.6 & $\begin{array}{l}\text { Dense microcolonies grown } \\
\text { on minimal media. Thin } \\
\text { phenotype. ITPG-induced } \\
\text { GFP cytosol marker. Time } \\
\text { lapse. Imaged by the Wiggins } \\
\text { lab. }\end{array}$ \\
\hline & & 141 & 4536 & 4395 & 9.3 & $\begin{array}{l}\text { Dense microcolonies on LB. } \\
\text { Time lapse. Imaged by the } \\
\text { Wiggins lab. } \\
\end{array}$ \\
\hline & & 2 & 2277 & - & - & $\begin{array}{l}\text { Treatment with cephalexin. } \\
\text { Tn7::GFP. Imaged by the } \\
\text { Mougous lab. }\end{array}$ \\
\hline & $\begin{array}{l}\text { CS703- } \\
1^{62}\end{array}$ & 80 & 23169 & 1299 & 2.6 & $\begin{array}{l}\text { Mutant grown on LB and } \\
\text { aztreonam. Elongated and } \\
\text { branching phenotypes. Time } \\
\text { lapse. Imaged by the } \\
\text { Mougous lab. }\end{array}$ \\
\hline \multirow[t]{2}{*}{ Shigella flexneri } & \multirow[t]{2}{*}{ M90T } & 117 & 256618 & 1409 & 3.0 & $\begin{array}{l}\text { Treatment with A22. } \\
\text { Tn7::GFP. Frames selected } \\
\text { from time lapse after } 1 \mathrm{hr} \\
\text { growth. Imaged by the } \\
\text { Mougous lab. }\end{array}$ \\
\hline & & 6 & 4482 & 4318 & 9.2 & $\begin{array}{l}\text { Treatment with cephalexin. } \\
\text { Tn7::GFP. Frames selected } \\
\text { from time lapse after } 1 \mathrm{hr} \\
\text { growth. Imaged by the } \\
\text { Mougous lab. }\end{array}$ \\
\hline $\begin{array}{l}\text { Francisella } \\
\text { tularensis subsp. } \\
\text { novicida }\end{array}$ & U112 & 5 & 20166 & 496 & 1.1 & $\begin{array}{l}\text { Small and extremely low- } \\
\text { contrast cells. Tn7::GFP. } \\
\text { Imaged by the Mougous lab. } \\
\end{array}$ \\
\hline \multirow[t]{3}{*}{$\begin{array}{l}\text { Acinetobacter } \\
\text { baylyi }\end{array}$} & \multirow[t]{3}{*}{$\mathrm{ADP} 1^{63}$} & 2169 & 60601 & 3336 & 7.1 & $\begin{array}{l}\text { Deletion of essential gene } \\
\text { murA. Rounded phenotype. } \\
\text { Time lapse. Imaged by the } \\
\text { Wiggins lab. }\end{array}$ \\
\hline & & 241 & 1313 & 1133 & 2.4 & $\begin{array}{l}\text { Deletion of essential gene } \\
f t s N \text {. Filamentous phenotype. } \\
\text { Time lapse. Imaged by the } \\
\text { Wiggins lab. }\end{array}$ \\
\hline & & 540 & 10013 & 2227 & 4.7 & $\begin{array}{l}\text { Deletion of essential gene } \\
\text { dnaA. Filamentous } \\
\text { phenotype. Time lapse. } \\
\text { Imaged by the Wiggins lab. }\end{array}$ \\
\hline $\begin{array}{l}\text { Burkholderia } \\
\text { thailandensis }\end{array}$ & E264 ${ }^{64}$ & 30 & 62005 & 5122 & 10.9 & $\begin{array}{l}\text { Selected panels from a self- } \\
\text { intoxication experiment. } \\
\text { Cells exhibit internal } \\
\text { structure and low contrast in } \\
\text { microcolonies. Tn } 7:: \text { GFP. } \\
\text { Time lapse. Imaged by the } \\
\text { Mougous lab. } \\
\end{array}$ \\
\hline $\begin{array}{l}\text { Helicobacter } \\
\text { pylori }\end{array}$ & LHS100 ${ }^{50}$ & 15 & 13014 & - & - & $\begin{array}{l}\text { Helical phenotype. Grown, } \\
\text { fixed, and stained with } \\
\text { Alexaflour } 488 \text { in the lab of }\end{array}$ \\
\hline
\end{tabular}




\begin{tabular}{|c|c|c|c|c|c|c|}
\hline & & & & & & $\begin{array}{l}\text { Nina Salama. Imaged by the } \\
\text { Mougous lab. }\end{array}$ \\
\hline & & 19 & 1668 & 701 & 1.5 & $\begin{array}{l}\text { Treated with aztreonam. } \\
\text { Filamentous, helical } \\
\text { phenotype. Grown, fixed, and } \\
\text { stained with Alexaflour } 488 \\
\text { in the lab of Nina Salama. } \\
\text { Imaged by the Mougous lab. }\end{array}$ \\
\hline $\begin{array}{l}\text { Caulobacter } \\
\text { crescentus }\end{array}$ & NA1000 52 & 4 & 1787 & 756 & 1.6 & $\begin{array}{l}\text { Grown in HIGG media to } \\
\text { induce stalk phenotype. } \\
\text { Cultivation and imaging done } \\
\text { in the lab of Yves Brun. }\end{array}$ \\
\hline $\begin{array}{l}\text { Streptomyces } \\
\text { pristinaespiralis }\end{array}$ & $\begin{array}{l}\text { NRRL } \\
2958\end{array}$ & 17 & 2339 & 270 & 0.6 & $\begin{array}{l}\text { Grown on rich media to } \\
\text { induce filamentous } \\
\text { phenotype. Imaged by the } \\
\text { Mougous lab. } \\
\end{array}$ \\
\hline Vibrio cholerae & A1552 $2^{65}$ & 2 & 2627 & 2265 & 4.8 & $\begin{array}{l}\text { Cells have short but curved } \\
\text { morphology and form dense, } \\
\text { low-contrast microcolonies. } \\
\text { Tn } 7:: \text { GFP. Obtained from the } \\
\text { lab of Fitnat Yildiz. Imaged } \\
\text { in the Mougous lab. } \\
\end{array}$ \\
\hline $\begin{array}{l}\text { Serratia } \\
\text { proteamaculans } \\
\text { E. coli }\end{array}$ & $\begin{array}{l}568 \\
\mathrm{DH} 5 \alpha\end{array}$ & 43 & 100146 & 1244 & 2.6 & $\begin{array}{l}\text { 1:1 mixture. S.p. labelled via } \\
\text { Tn7::GFP, E.c. unlabeled. } \\
\text { Time lapse. Imaged in the } \\
\text { Mougous lab. }\end{array}$ \\
\hline $\begin{array}{l}\text { Pseudomonas } \\
\text { aeruginosa } \\
\text { Staphylococcus } \\
\text { aureus } \\
\end{array}$ & $\begin{array}{l}\text { PAO1 } \\
\text { USA300 }\end{array}$ & 3 & 2662 & 3688 & 7.8 & $\begin{array}{l}\text { 1:1 mixture. P.a. labelled via } \\
\text { Tn7::GFP, S.a. unlabeled. } \\
\text { Imaged in the Mougous lab. }\end{array}$ \\
\hline \multirow[t]{2}{*}{$\begin{array}{l}\text { P. aeruginosa } \\
\text { S. aureus } \\
\text { V. cholerae } \\
\text { Bacillus subtilis }\end{array}$} & \begin{tabular}{|l|} 
PAO1 \\
USA300 \\
A1552 \\
HM1350
\end{tabular} & 21 & 33281 & 4678 & 9.9 & $\begin{array}{l}\text { 1:1:1:1 mixture. P.a. and } V . c . \\
\text { labelled via Tn7::GFP, S.a. } \\
\text { and B.s. labelled with red } \\
\text { membrane dye. Imaged in the } \\
\text { Mougous lab. }\end{array}$ \\
\hline & & 4833 & 700904 & 47070 & 100 & \\
\hline
\end{tabular}

